ARTICLE

https://doi.org/10.1038/s41467-019-08590-7

\title{
tmem33 is essential for VEGF-mediated endothelial calcium oscillations and angiogenesis
}

\author{
Aaron M. Savage ${ }^{1,2}$, Sathishkumar Kurusamy ${ }^{3}$, Yan Chen ${ }^{1,2}$, Zhen Jiang ${ }^{1,2}$, Karishma Chhabria 1,2, \\ Ryan B. MacDonald ${ }^{1,2}$, Hyejeong R. Kim ${ }^{2,4}$, Heather L. Wilson 1,2, Fredericus J.M. van Eeden²,4,5, \\ Angel L. Armesilla ${ }^{3}$, Timothy J.A. Chico ${ }^{1,2}$ \& Robert N. Wilkinson (10) 1,2,5
}

\begin{abstract}
Angiogenesis requires co-ordination of multiple signalling inputs to regulate the behaviour of endothelial cells (ECS) as they form vascular networks. Vascular endothelial growth factor (VEGF) is essential for angiogenesis and induces downstream signalling pathways including increased cytosolic calcium levels. Here we show that transmembrane protein 33 (tmem33), which has no known function in multicellular organisms, is essential to mediate effects of VEGF in both zebrafish and human ECs. We find that tmem33 localises to the endoplasmic reticulum in zebrafish ECs and is required for cytosolic calcium oscillations in response to Vegfa. tmem33-mediated endothelial calcium oscillations are critical for formation of endothelial tip cell filopodia and EC migration. Global or endothelial-cell-specific knockdown of tmem33 impairs multiple downstream effects of VEGF including ERK phosphorylation, Notch signalling and embryonic vascular development. These studies reveal a hitherto unsuspected role for tmem33 and calcium oscillations in the regulation of vascular development.
\end{abstract}

\footnotetext{
${ }^{1}$ Department of Infection, Immunity and Cardiovascular Disease, Medical School, University of Sheffield, Sheffield, UK. ${ }^{2}$ The Bateson Centre, University of Sheffield, Sheffield, UK. ${ }^{3}$ Cardiovascular Molecular Pharmacology Laboratory, Faculty of Science and Engineering, Research Institute in Healthcare Science, School of Pharmacy, University of Wolverhampton, Wolverhampton, UK. ${ }^{4}$ Department of Biomedical Science, University of Sheffield, Sheffield, UK.

${ }^{5}$ Sheffield CRISPRi Facility, Sheffield, UK. These authors contributed equally: Timothy J. A. Chico, Robert N. Wilkinson. Correspondence and requests for materials should be addressed to R.N.W. (email: Robert.wilkinson@sheffield.ac.uk)
} 
T he formation of a complex vascular network is an essential process during embryonic development, which is vital for growth of tissues and is frequently dysregulated during disease in the adult. Endothelial cells (ECs) line the inner lumen of blood vessels and their organisation into complex branching networks requires co-ordination of molecular outputs coupled to specific cellular behaviours via a process primarily orchestrated by signalling from vascular endothelial growth factor (VEGF) ${ }^{1}$.

VEGF is a morphogen that signals via different ligands to induce motile and invasive behaviour, which drives blood vessel sprouting. VEGFA primarily controls angiogenesis from arteries via its cognate receptor VEGFR2/KDR, whereas VEGFC promotes sprouting from veins via VEGFR3/FLT42 ${ }^{2}$. Migrating ECs extend filopodia to sense VEGF signals via $K d r$ (VEGFR2), as they form a new sprouting vessel ${ }^{3}$. Leading angiogenic ECs are termed tip cells, which upregulate dll4 transcription, inducing Notch signalling in neighbouring cells, and this acts to limit excessive angiogenic sprouting ${ }^{4}$. Neighbouring Notch-expressing cells join the sprout as stalk cells, which in zebrafish tend to exhibit reduced proliferative capacity compared with tip cells ${ }^{4,5}$.

VEGFA has been shown to promote proliferation of ECs in vitro via VEGFR2-mediated activation of the RAS/RAF/ERK pathway without affecting migration ${ }^{6}$. Others, however, have shown that inhibition of ERK phosphorylation in vivo inhibits EC migration but not proliferation during angiogenesis ${ }^{7}$. ERK activation is induced via PLCG1 phosphorylation in vitro ${ }^{8}$, which generates inositol 1,4,5-trisphosphate (IP3). IP3 subsequently activates inositol triphosphate receptor (IP3R) $\mathrm{Ca}^{2+}$ channels within the endoplasmic reticulum (ER) to increase cytosolic $\mathrm{Ca}^{2+}$ concentrations and activate protein kinase $\mathrm{C}$ to phosphorylate ERK $^{9}$. ERK activation is required to promote angiogenesis and has been shown to promote expression of tip cell markers including $d l l 4^{7,10}$ and $f l t 4^{7}$. VEGF and Notch therefore balance formation of tip and stalk cells within developing blood vessels and modulate the relative migration and proliferation of ECs during angiogenesis ${ }^{11} . \mathrm{Ca}^{2+}$ is a universal secondary messenger, which achieves specificity using complex signalling modalities. These include encoding information to activate cellular responses within cytosolic $\mathrm{Ca}^{2+}$ oscillations ${ }^{12}$. How EC $\mathrm{Ca}^{2+}$ oscillations integrate complex molecular outputs to precisely control discrete cellular behaviours in a developing vascular network remains unknown.

ECs are generally thought to be non-excitable and as such utilise store-operated calcium entry (SOCE) as their primary means to maintain $\mathrm{Ca}^{2+}$ levels in the ER after influx ${ }^{13,14}$. The principal trigger for SOCE activation occurs when intracellular calcium stores with the ER are depleted and these are refilled via interaction of TRPC1 and ORAI1 on the plasma membrane with ER-resident STIM1, which form the calcium-release activated channel ${ }^{15-18}$. SOCE relies on modulation of the actin cytoskeleton, which requires $\mathrm{Ca}^{2+}$-dependent proteins for ER motility ${ }^{19}$.

We have identified transmembrane protein 33 (TMEM33) as a component of the $\mathrm{ER} \mathrm{Ca}^{2+}$ signalling machinery required for angiogenesis. TMEM33 is a three-pass transmembrane domain protein conserved throughout evolution with two paralogues in the budding yeast Saccharomyces cerevisiae, Pom33 and Per33, showing enrichment in the nuclear pore and ER, respectively ${ }^{20,21}$. In the fission yeast Schizosaccharomyces pombe, the TMEM33 orthologue Ttsip contributes to maintenance of the cortical ER network $^{22}$. Human TMEM33 has been shown to localise to the nuclear envelope and ER in vitro, where it has been suggested to regulate the tubular structure of the ER by suppressing the membrane-shaping activity of reticulons ${ }^{23,24}$. However, its function within the ER in multicellular organisms remains unknown.

Here we describe the first characterisation of tmem33 in a multicellular organism and show that tmem 33 is required in an
EC-specific manner for Vegfa-mediated $\mathrm{Ca}^{2+}$ oscillations, to promote angiogenesis in zebrafish embryos. The requirement for tmem 33 during the response to VEGF is conserved from zebrafish to humans. Furthermore, tmem33-mediated endothelial $\mathrm{Ca}^{2+}$ oscillations are critical for formation of endothelial filopodia and contribute to activation of ERK and induction of Notch signalling to co-ordinate vascular morphogenesis.

\section{Results}

tmem33 knockdown impairs vascular and pronephric development. We find tmem33 is expressed ubiquitously during zebrafish segmentation (Fig. 1a-c) and by $26 \mathrm{~h}$ post fertilisation (hpf) is enriched in the trunk vasculature and pronephros (Fig. 1d). TMEM33 expression has been previously identified within the nuclear envelope and ER in human cells $s^{23,24}$. We expressed a fulllength C-terminal tmem33-EGFP fusion messenger RNA in developing zebrafish embryos and found Tmem33-EGFP fusion protein to localise to structures indicative of nuclear envelope (Fig. 1e-g, blue arrowheads) and ER (Fig. 1e-g, white arrowheads) of ECs within the caudal artery.

To knock down tmem 33 expression and establish its function during embryonic development, we next used two splice blocking morpholinos targeting exon 3, (Supplementary Fig. 1a, b, Supplementary Tables 1 and 2). All experiments were conducted using co-injection of both morpholinos. tmem33 morphants exhibited reduced segmental artery (SeA) length compared with controls (Fig. 1h-k, white arrowheads, p). By $30 \mathrm{hpf}$, SeAs in control morphants had reached the dorsal roof of the neural tube and begun to sprout laterally to form the dorsal longitudinal anastomotic vessel (DLAV) (Fig. 1h, i yellow arrowhead), whereas tmem 33 morphants often displayed abnormal spade-shaped tip cell morphology (Fig. 1k, white arrowhead) and were observed to stall at the level of the horizontal myoseptum. Consistent with this, tmem33 morphants displayed reduced SeA migration rate (Supplementary Fig. 2a-d, yellow arrowheads, Supplementary Fig. 2e). Expression of an arterial marker $\operatorname{Tg}(0.8 f l t 1: R F P)^{25}$ in tmem33 morphants was indistinguishable from controls and restricted to the dorsal aorta (DA) and SeAs from day 1, suggesting the DA was correctly specified in tmem 33 morphants (Fig. 11, n). Onset of circulation and blood flow was normal in tmem33 morphants (Supplementary Fig. 3 and Supplementary Movies 1-3). Despite this early delay in sprouting angiogenesis, by $48 \mathrm{hpf}$, most SeAs in tmem 33 morphants had completed their dorsal migration, but many failed to migrate laterally, resulting in a primitive and discontinuous DLAV (Fig. 1l-o, yellow arrowheads, q). In addition, tmem33 morphants displayed absent parachordal lymphangioblasts (Fig. 1l, n, red arrowheads). The thoracic duct (TD) is the first major lymphatic vessel to develop in zebrafish ${ }^{26}$ and forms by ventral migration of parachordal lymphangioblasts. In keeping with the absence of parachordal lymphangioblasts, the TD was absent in tmem 33 morphants (Fig. 1r, s, asterisks).

tmem33 knockdown reduces $\mathrm{EC} \mathrm{Ca}^{2+}$ oscillations and filopodia. VEGF signalling is essential for SeA formation in zebrafish $^{7,27-31}$ and lymphatic development ${ }^{26,32-36}$. Although TMEM33 has never been implicated in angiogenesis or VEGF signalling, the similarity of the phenotype of embryos with lossof-function of tmem33 or VEGF signalling led us to hypothesise that tmem 33 may regulate VEGF signalling in ECs. Furthermore, as VEGF increases EC intracellular $\mathrm{Ca}^{2+37}$, which is dependent upon the interaction of plasma membrane proteins with ERresident proteins via $S O C E^{16,38}$, we also postulated that tmem33 may regulate VEGF-induced $\mathrm{EC} \mathrm{Ca}^{2+}$ signalling, given its indicative expression in the ER (Fig. 1e-g). 


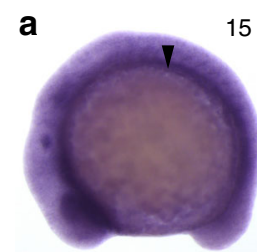

C

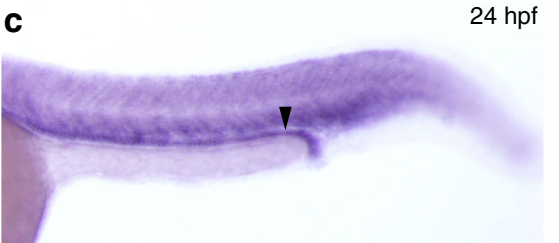

d

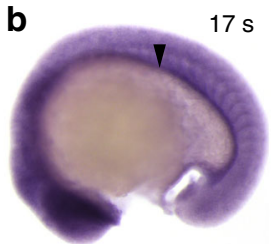

$24 \mathrm{hpf}$
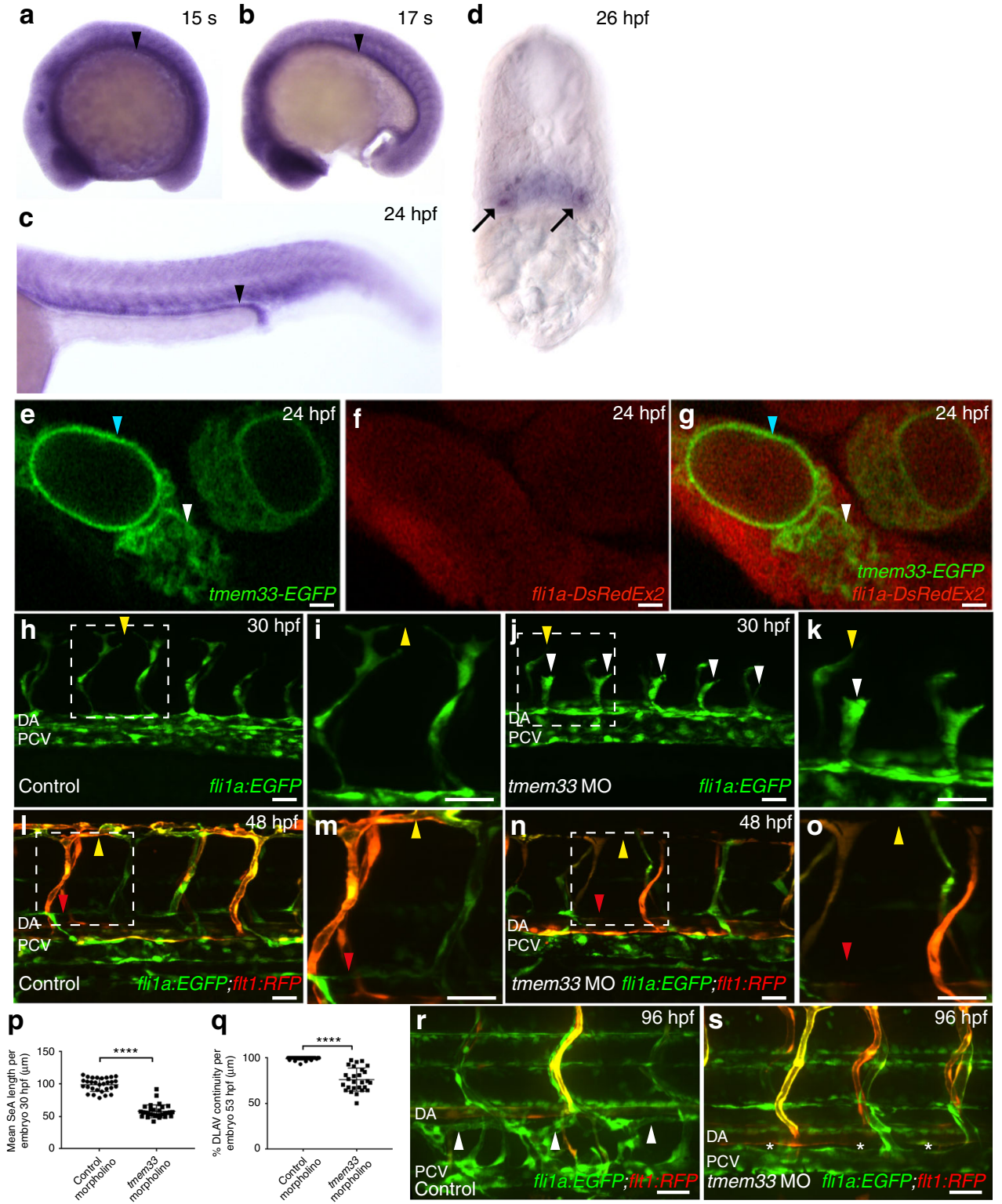

Fig. 1 tmem33 knockdown inhibits angiogenesis and localises to the ER in ECs. a-d tmem33 is expressed ubiquitously during segmentation, but displays enrichment in the pronephros (black arrowheads) and somite boundaries, which is more pronounced from 24 hpf. Pronephric expression is evident in 26 hpf transverse sections (black arrows). e-g Tmem33-EGFP protein localises to the nuclear envelope (blue arrowheads) and ER (white arrowheads) within the caudal artery in fliTa:DsRedEx2 embryos (Scale bars $1 \mu \mathrm{m}$ ). $\mathbf{h}-\mathbf{k}$ tmem33 morphants injected with $0.4 \mathrm{ng}$ morpholinos display delayed migration of $\mathrm{Tg}$ (fli7a:egfp) positive SeAs, which stall at the horizontal myoseptum (j, $\mathbf{k}$, white arrowheads), compared with control $T g(f l i 1 a$ :egfp) positive morphants (h, i), which begin to anastomose by $30 \mathrm{hpf}$ (yellow arrowheads) (scale bars $50 \mu \mathrm{m}$ ). I-o By 48 hpf, Tg(flita:EGFP;-0.8flt7:RFP) tmem33 morphant SeAs complete dorsal migration, but display incomplete DLAV formation (n, $\mathbf{0}$, yellow arrowheads) and lack lymphatic vasculature (red arrowheads). At $48 \mathrm{hpf} T g$ (flita: EGFP; - 0.8flt1:RFP) control morphants display secondary angiogenesis (I, $\mathbf{m}$, yellow arrowheads) and parachordal lymphangioblasts (red arrowhead) (scale bars $50 \mu \mathrm{m})$. p tmem33 morphants injected with $0.4 \mathrm{ng}$ morpholinos display reduced SeA length at $30 \mathrm{hpf}\left(t\right.$-test ${ }^{\star \star \star \star} p=<0.0001 ; t=4.075 ; \mathrm{DF}=24$. $n=3$ repeats, 10 embryos per group). q tmem33 morphants injected with $0.4 \mathrm{ng}$ morpholinos display incomplete formation of DLAV ( $t$-test ${ }^{\star \star \star \star} p=<$ $0.0001 ; t=5.618 ; \mathrm{DF}=28 . n=3$ repeats, 9 or 10 embryos per group). $\mathbf{r}, \mathbf{s}$ Thoracic duct formation is impaired in tmem33 morphants injected with 0.4 ng morpholinos (white asterisks), compared with control morphants (white arrowheads) (scale bars $50 \mu \mathrm{m}$ ). DA, dorsal aorta; PCV, posterior cardinal vein. Source data are provided as a Source Data file

To determine the effect of tmem 33 knockdown on EC calcium signalling in vivo, we generated an endothelial $\mathrm{Ca}^{2+}$ reporter line $\operatorname{Tg}\left(f l i 1 a: g a l 4 F^{u b s 3} ;\right.$ uas-gCaMP7ash392), hereafter referred to as flila:gal4FFubs3; uas-GCaMP7a. Recently, a similar transgenic has been employed as an indirect readout of VEGF signalling activity in zebrafish $\mathrm{ECs}^{39}$. To first establish whether fli1a:gal4FFubs3; uasGCaMP7a embryos reliably reported fluctuations in endothelial $\mathrm{Ca}^{2+}$ signalling, we treated embryos with Thapsigargin to inhibit the sarcoplasmic or ER Ca-ATPase (SERCA) family of $\mathrm{Ca}^{2+}$ pumps ${ }^{40}$ and thereby raise endothelial $\mathrm{Ca}^{2+}$ levels. Indeed, Thapsigargin treatment significantly increased GCaMP7a fluorescence in tip cells (Supplementary Fig. 4a,b,d) and reduced cytosolic $\mathrm{Ca}^{2+}$ oscillations in tip cells (Supplementary Fig. 4e). Conversely, as VEGF-mediated elevations in cytosolic $\mathrm{Ca}^{2+}$ are dependent upon IP3R function ${ }^{37}$, we treated embryos with an IP3R antagonist 2-APB, to reduce cytosolic $\mathrm{Ca}^{2+}$ (Supplementary 

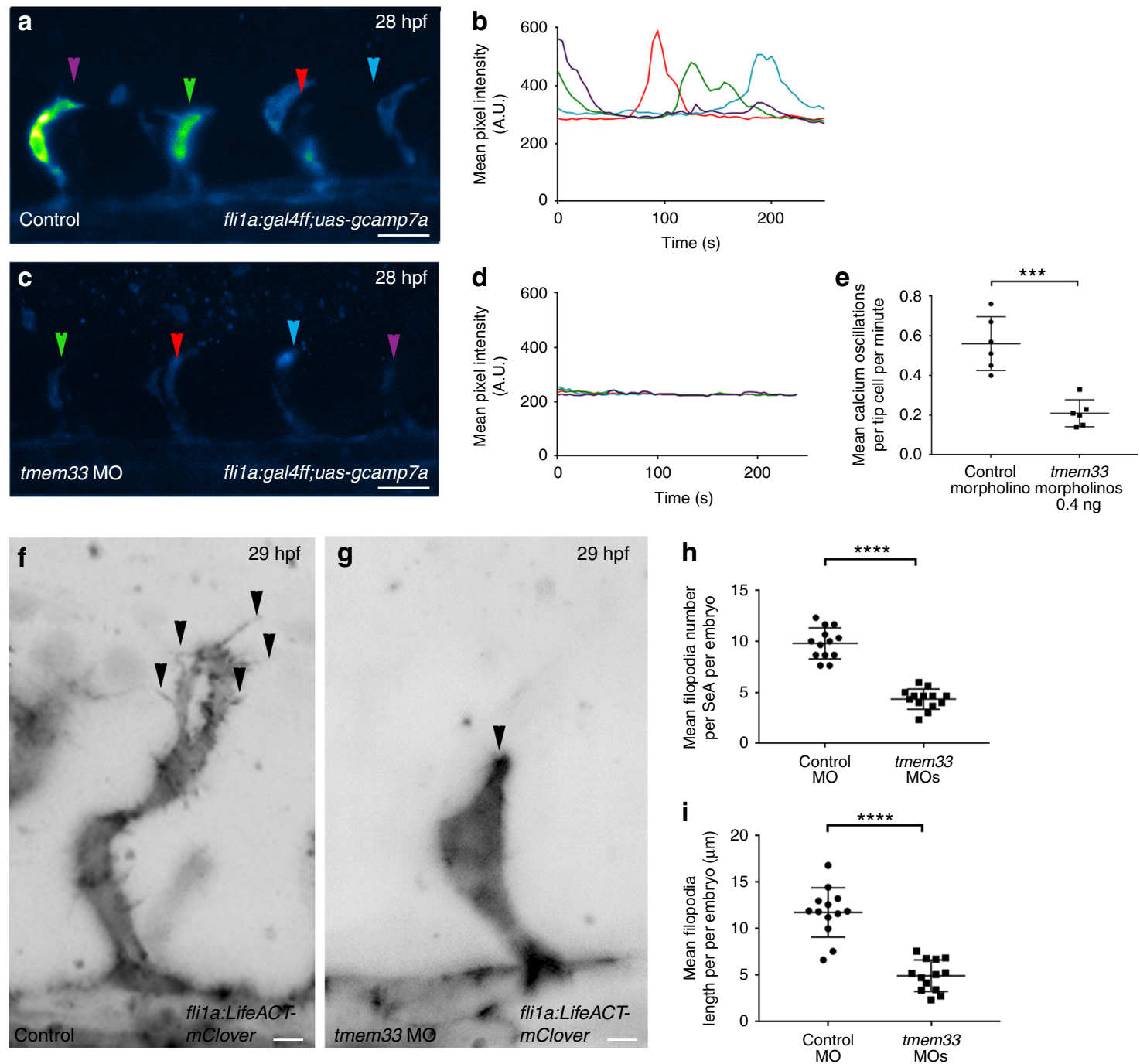

Fig. 2 tmem33 knockdown reduces $\mathrm{Ca}^{2+}$ oscillations in tip cells and reduces EC filopodia. a-d tmem33 morphants injected with 0.4 ng morpholinos display reduced endothelial $\mathrm{Ca}^{2+}$ oscillations. Intensity projections show $\mathrm{Ca}^{2+}$ oscillations in both control and tmem33 morphants, highlighting intensity over duration of the time lapse. Change in fluorescence over $300 \mathrm{~s}$ for four SeAs, trace colours correspond to equivalent arrowheads (scale bars $50 \mu \mathrm{m}$ ). e tmem33 morphants display a significant reduction in $\mathrm{Ca}^{2+}$ oscillations when compared with control morphants (unpaired $t$-test ${ }^{\star \star \star \star} p=<0.001 ; t=$ 5.633; DF $=10,2$ repeats, $n=3$ embryos per group). $\mathbf{f}, \mathbf{g}$ tmem33 morphants injected with $0.4 \mathrm{ng}$ morpholinos display delayed migration of SeAs, which extend fewer filopodia, compared with controls (black arrowheads) (scale bars $5 \mu \mathrm{m}$ ). $\mathbf{h}$ tmem33 morphants display significantly fewer filopodia per SeA compared with controls. Measurements taken from $n=4$ SeAs per embryo. Unpaired $t$-test, ${ }^{\star \star \star \star} p=\langle 0.0001 ; t=7.805 ; D F=24,2$ repeats, $n=6$ or 7 embryos per group). i tmem33 morphants display reduced filopodia length compared with controls, measurements taken from $n=4$ SeAs. Unpaired $t$-test ${ }^{\star \star \star \star} p<0.0001 ; t=10.86 ; \mathrm{DF}=24 ; 2$ repeats, $n=6$ or 7 embryos per group. Source data are provided as a Source Data file

Fig. 4a,c). fli1a:GCaMP7a embryos treated with 2-APB displayed reduced EC GCaMP7a fluorescence and reduced frequency of $\mathrm{Ca}^{2+}$ oscillations in tip cells (Supplementary Fig. 4a,c,d,e).

Having established fli1a:gal4FF ${ }^{u b s}$; uas-GCaMP7a as a reporter of EC cytosolic $\mathrm{Ca}^{2+}$ levels in vivo, we examined the effect of tmem33 knockdown on EC $\mathrm{Ca}^{2+}$ oscillations. tmem33 knockdown in fli1a:gal4FFubs3;uas-GCaMP7a embryos significantly reduced the frequency of $\mathrm{Ca}^{2+}$ oscillations and GCaMP7a fluorescence intensity in EC tip cells (Fig. 2a-e and Supplementary Movies 4,5). Furthermore, treatment of flila:gal4FFubs3; uas-GCaMP7a embryos with the VEGFR inhibitor Tivozanib/ AV951 reduced $\mathrm{Ca}^{2+}$ oscillations in a similar manner to tmem33 knockdown (Supplementary Fig. 5a-d,i). In contrast, $N$-[N-(3,5-Difluorophenacetyl)-L-alanyl]-S-phenylglycine t-butyl ester (DAPT)-mediated inhibition of Notch signalling, which negatively regulates VEGF signalling ${ }^{41}$, significantly increased cytosolic $\mathrm{Ca}^{2+}$ oscillations within EC tip cells (Supplementary
Fig. 5e,f,i). These data indicate tmem33 knockdown reduced endothelial

$\mathrm{Ca}^{2+}$ activity to a similar level as VEGF inhibition and are consistent with studies that demonstrate EC calcium signalling mediates the response to VEGF in vivo ${ }^{39}$.

The delayed SeA sprouting, abnormal tip cell morphology and aberrant DLAV anastomosis following tmem33 knockdown (Fig. 1h-o) are similar to phenotypes observed following inhibition of filopodia formation in endothelial tip cells $\mathrm{s}^{42}$. Knockdown of tmem33 in Tg(fli1a:lifeACT-mClover) ${ }^{\text {sh } 467}$, which labels filamentous actin in ECs, substantially reduced the number and length of filopodia present on EC tip cells (Fig. 2f-i), suggesting defective filopodia formation may contribute to impaired angiogenesis in tmem33 morphants. As filopodia are known to express VEGF receptors and transduce the migratory signal upon VEGF ligand binding ${ }^{3,43}$, we examined whether loss of filopodia could account for the reduction in $\mathrm{EC} \mathrm{Ca}^{2+}$ 
oscillations observed in tmem33 morphants (Fig. 2a-e, Supplementary Movies 4,5). Tg(fli1a:lifeACT-mClover) embryos treated with the actin depolymerising agent Latrunculin B did not form filopodia (Supplementary Fig. 6a-c, black arrowheads), instead forming rapidly depolymerising F-actin foci in keeping with previous reports ${ }^{42}$. Treatment of fli1a:gal4FFubs3; uas-GCaMP7a embryos with Latrunculin B had no significant effect on frequency of endothelial $\mathrm{Ca}^{2+}$ oscillations in tip cells in comparison with controls (Supplementary Fig. 6d-f, white arrowheads). This suggests loss of EC filopodia in tmem33 morphants is not responsible for reduced $\mathrm{EC} \mathrm{Ca}^{2+}$ oscillations.

tmem33 mutant zebrafish embryos display genetic compensation. As morpholino knockdown is transient and may induce offtarget effects, we next generated zebrafish tmem 33 mutants using transcription activator-like effector nucleases (TALENs) ${ }^{44}$. We identified a mutant allele (hereafter referred to as sh443) with a 2 bp deletion in exon 3, which induces a premature stop codon within exon 4 encoding the second conserved transmembrane helix (Supplementary Fig. 7a,b). tmem $33^{\text {sh } 443}$ mutants display substantially reduced expression of tmem 33 mRNA (Supplementary Fig. 7c-e) likely via nonsense-mediated decay, indicating this mutation represents a severe loss-of-function or null allele. However, in contrast to tmem33 morphants, vascular (Supplementary Fig. 7f,g) and pronephric development was normal in tmem $33^{\text {sh } 443}$ mutants and homozygous tmem 33 mutant adults were viable. Recent studies describe mechanisms by which the zebrafish genome can compensate for genetic mutations but not morpholino knockdown ${ }^{45}$. Consistent with such compensation, homozygous tmem $33^{\text {sh } 443}$ mutants injected with tmem 33 morpholinos titrated to a level, which did not significantly induce $p 53$ expression (Supplementary Fig. 7h), displayed significantly longer SeAs compared with heterozygote or wild-type embryos (Supplementary Fig. 7i-1, arrowheads). In addition to the described effect on vascular and lymphatic development, tmem33 morphants exhibited increased glomerular size (Supplementary Fig. $7 \mathrm{~m}-\mathrm{o}$, arrows) and homozygous tmem $33^{\text {sh } 443}$ mutants were protected against the effect of the tmem33 morpholino on glomerular size (Supplementary Fig. $7 \mathrm{~m}-\mathrm{o}$, arrows). These data suggest tmem $33^{\text {sh } 443}$ mutants exhibit genetic compensation or transcriptional adaptation ${ }^{46}$.

tmem33 crispant embryos phenocopy tmem33 morphant embryos. As tmem 33 mutants appeared to display genetic compensation, and in order to confirm the morpholino-induced phenotype, we next knocked down tmem33 using Clustered Regularly Interspaced Short Palindromic Repeats interference (CRISPRi). This acts via steric inhibition of transcription ${ }^{47}$ and has been previously successful in zebrafish embryos ${ }^{45}$. In comparison with co-injection of mRNA encoding a catalytically inactive Cas9 nuclease (dCas9) ${ }^{47}$ and control single-guide RNAs (sgRNAs) lacking the dCas9 binding motif (Supplementary Table 2), co-injection of sgRNAs flanking the start codon of tmem33 with dCas9 mRNA into zebrafish embryos (hereafter referred to as crispants) reduced tmem 33 expression by in situ hybridisation (Supplementary Fig. 8a,b, arrowhead) and quantitative reverse-transcriptase PCR (qRT-PCR) (Supplementary Fig. 8c). tmem 33 CRISPRi significantly reduced $\mathrm{Ca}^{2+}$ oscillations in tip cells (Supplementary Fig. 8d-h) and endothelial filopodia number and length (Supplementary Fig. 8i-l, arrowheads). We were able to rescue the angiogenic defects in tmem 33 morphants and crispants by co-injection of full-length tmem33 mRNA (Supplementary Fig. 9) confirming that these were due to reduced expression of tmem33. Interestingly, at minimal phenotypic doses, induction of p53 expression was substantially reduced in tmem33 crispants in comparison with tmem 33 morphants injected with $4 \mathrm{ng}$ morpholino (Supplementary Fig. $8 \mathrm{~m}$ ), suggesting CRISPRi may be less subject to nonspecific effects than morpholinos. Taken together, these data demonstrate that transcriptional inhibition of tmem33 via CRISPRi induces endothelial defects highly similar to those generated by morpholino-mediated translational inhibition of tmem33 (Fig. 2). These data indicate tmem33 is required for endothelial cytosolic $\mathrm{Ca}^{2+}$ oscillations, endothelial filopodia formation and normal angiogenesis.

tmem33 promotes angiogenesis in an EC-specific manner. The morphant and crispant phenotypes described above were induced by global translational/transcriptional inhibition and tmem33 is expressed in tissue other than ECs and the developing kidney. We therefore sought to examine the effect of EC- and pronephrosspecific inhibition of tmem33. As CRISPRi employs a genetically encoded catalytically inactive Cas 9 nuclease (dCas9) 47 , we postulated that co-expressing $d \operatorname{Cas} 9$ under the control of a tissuespecific promoter and gene targeting sgRNAs may facilitate tissue-specific knockdown of tmem33. We therefore generated fli1a:dCas9, cryaa:cfp and enpep:dCas9, cryaa:cfp constructs (Supplementary Fig. 10a,b) that drive transient dCas9 expression under control of either EC or pronephros-specific promoters, respectively. These constructs were injected alongside Tol2 mRNA and gene-targeting sgRNAs to elicit tissue-specific gene knockdown.

In comparison with co-injection of dCas9 mRNA and control sgRNAs lacking the dCas9-binding motif (Supplementary Fig. 10c, arrowheads, g arrows, $k, 1)$, co-injection of sgRNAs targeting tmem 33 and $d$ Cas 9 mRNA into progeny of an intercross between $\mathrm{Tg}(\text { flila:AC-TagRFP })^{\text {sh } 511 /+}$ and $\mathrm{Tg}(w t 1 b: e g f p) /+$ fish induced both abnormal vascular and pronephric development (Supplementary Fig. 10d, arrowheads h, arrows k, l,) as previously demonstrated. In contrast, embryos injected with tmem33 sgRNAs and flila:dCas9, cryaa:cfp construct displayed significantly reduced DLAV continuity but normal kidney development (Supplementary Fig. 10e, arrowheads i, arrows k, l), whereas embryos injected with tmem33 sgRNAs and enpep:dCas9, cryaa: $c f p$ construct, restricting CRISPRi knockdown to the kidney, developed distended glomeruli, whereas the vasculature was normal (Supplementary Fig. 10f, arrowheads j, arrows k, l). We next generated a stable transgenic line expressing dCas9 in EC $\mathrm{Tg}$ (fli1a:dCas9, cryaa-cerulean) $)^{\text {sh512}}$, hereafter referred to as $\operatorname{Tg}($ flila: dCas 9 ). To avoid the potential for reduced of knockdown efficiency by fluorescent tagging, we employed untagged dCas $9^{45}$. We screened for embryos expressing dCas 9 in ECs by in situ hybridisation (Fig. 3a-c) and validated EC-restricted expression of dCas9 using immunohistochemistry (Fig. 3d-g). As coinjection of dCas9 mRNA and control sgRNAs were consistent with normal vascular and pronephric development (Supplementary Fig. 8, S10), to test efficacy of EC gene knockdown in stable $d$ Cas 9 expressing transgenics, we first injected progeny of outcrosses from $\operatorname{Tg}($ flila:dCas 9$) /+\times T g(f l i 1 a: E G F P) /+$ with an sgRNA targeting dll4 (Fig. $3 \mathrm{~h}-\mathrm{j}$ ). Injection of sgRNA therefore served as an internal control in Cerulean Fluorescent Protein (CFP)-negative embryos. CFP-positive embryos displayed significantly increased DLAV diameter in comparison with CFPnegative embryos (Fig. 3h,i, arrowheads, j), consistent with previously observed ectopic DLAV sprouting in dll4 morphants and mutants ${ }^{48}$. Injection of tmem33 sgRNAs into $\operatorname{Tg}($ fli1a:dCas9) embryos delayed SeA sprouting (Fig. 3k-m, compare white arrowheads with yellow arrowheads) and induced discontinuous DLAV formation (Fig. 3n-p, yellow arrowheads) only in CFPpositive embryos, similar to tmem33 morphants (Fig. 1h-o) and crispants (Supplementary Fig. 10d,e, arrowheads, k). 

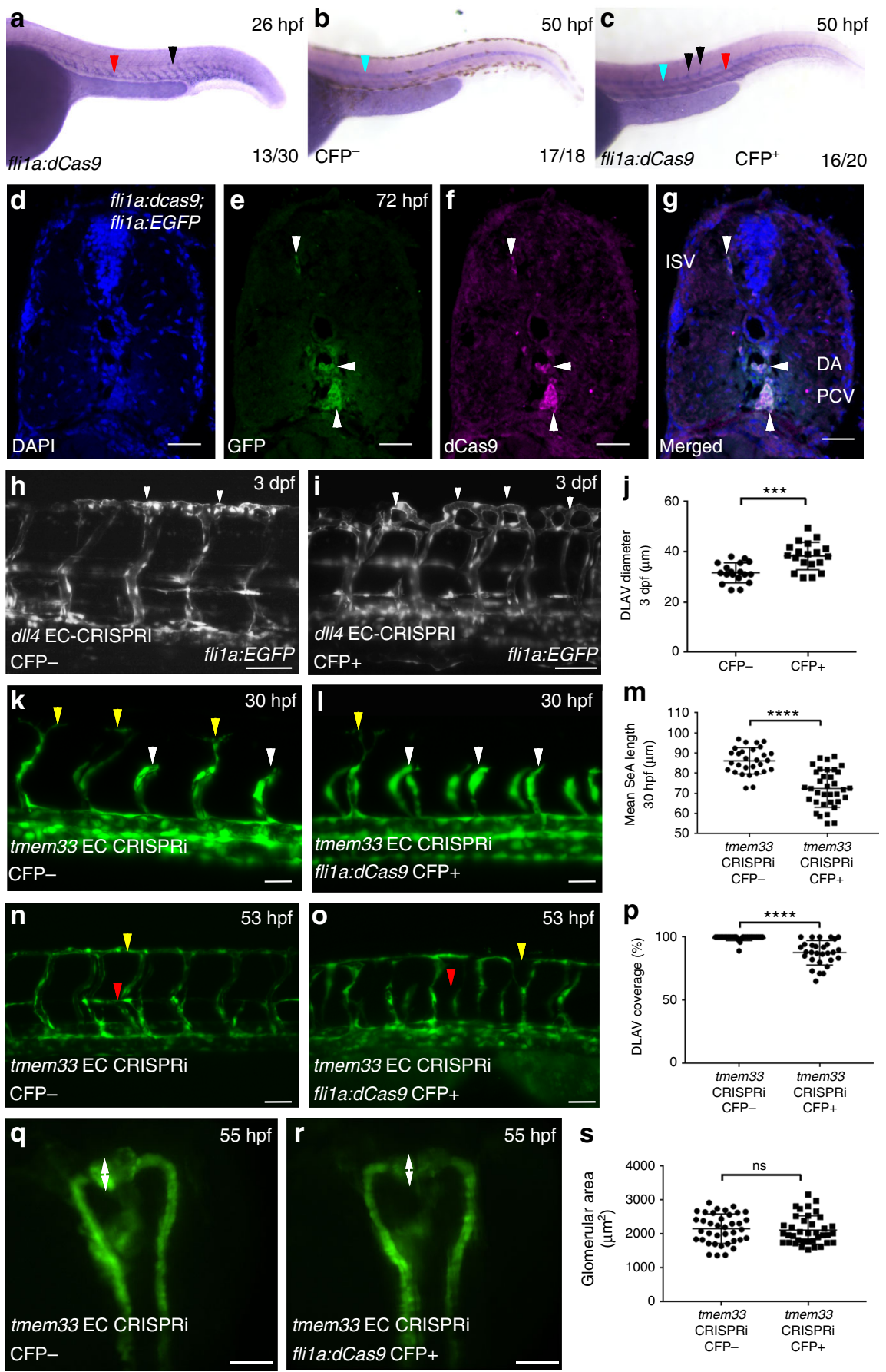

Fig. 3 Injection of sgRNAs targeting tmem33 into stable Tg(fli7a:dCas9;cryaa:CFP)sh512 embryos recapitulates knockdown of tmem33. a dCas9 is expressed within the developing vasculature (red arrowhead) including SeAs (black arrowheads) in 50\% of progeny from a $\mathrm{Tg}(f l i 7 a: d C a s 9 ; \mathrm{cryaa}$ :Cerulean)sh512/ + outcross at $26 \mathrm{hpf}$. At this stage the dominant cryaa:Cerulean marker is not expressed. $\mathbf{b} d$ Cas 9 is not expressed within the developing vasculature in CFP- $\mathrm{Tg}$ (fli1a:dCas9; cryaa:Cerulean) ${ }^{\text {sh512 }}$ transgenic embryos at 50 hpf. Probe trapping in notochord is highlighted (blue arrowhead). c $d$ Cas 9 is expressed within the dorsal aorta (red arrowhead) and SeAs (black arrowheads) in CFP+ Tg(fli7a:dCas9; cryaa:Cerulean)sh512 transgenic embryos at 50 hpf. Probe trapping in notochord is highlighted (blue arrowhead). $\mathbf{d}-\mathbf{g}$ Colocalisation of dCas9 and GFP in 72 hpf fli7a:dCas9; fli7a:EGFP embryo indicates EC restricted expression of dCas9 (white arrowheads) Scale bars $20 \mu \mathrm{m}$. h, i Tg(fli7a:dCas9; cryaa:Cerulean) embryos injected with sgRNA targeting dll4 phenocopies ectopic vascular looping within the DLAV previously observed in dII4 morphants and mutants at $3 \mathrm{dpf}$ (white arrowheads). $\mathbf{j}$ Tg(fliTa:dCas9;cryaa:Cerulean)-positive embryos injected with sgRNAs targeting dll4 display increased DLAV diameter (unpaired $t$-test ${ }^{\star \star \star} p=<0.001 ; t=4.203$ DF $=35 ; 2$ repeats; $n=9$ embryos per group). Scale bars $50 \mu \mathrm{m}$. k-m Tg(fli7a:dCas9; cryaa:Cerulean) embryos injected with sgRNAs targeting tmem33 display reduced SeA length (yellow arrowheads highlight normal SeAs, white arrowheads highlight delayed SeAs) at 30 hpf (unpaired $t$-test ${ }^{\star \star *} p=<0.0001 ; t=6.716$ DF $=62 ; 3$ repeats; $n=$ 9-12 embryos per group). Scale bars $50 \mu$ m. n-p Tg(fli7a:dCas9; cryaa:Cerulean) embryos injected with sgRNAs targeting tmem33 display absent parachordal lymphangioblasts (red arrowhead) and reduced DLAV continuity (yellow arrowheads) (unpaired $t$-test ${ }^{\star \star \star} p=<0.0001 ; t=6.399 \mathrm{DF}=56 ; 3$ repeats; $n=$ 9-11 embryos per group). Scale bars $50 \mu \mathrm{m}$. q-s Tg(fli7a:dCas9; cryaa:Cerulean) embryos injected with sgRNAs targeting tmem33 display no significant difference in glomerular area, compared with Tg(fli7a:dCas9;cryaa:Cerulean)-negative siblings embryos (unpaired $t$-test, ns $=$ not significant; $t=0.4048$; $\mathrm{DF}=74 ; 3$ repeats; $n=12-13$ embryos per group). Scale bars $200 \mu \mathrm{m}$. Source data are provided as a Source Data file 


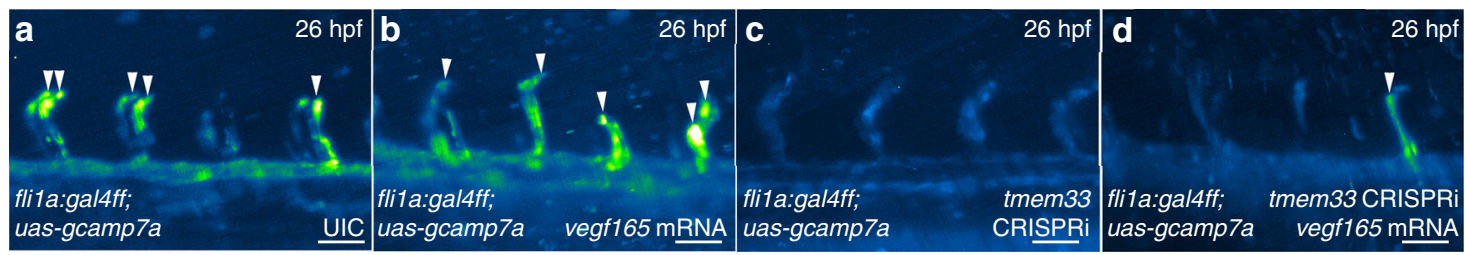

e
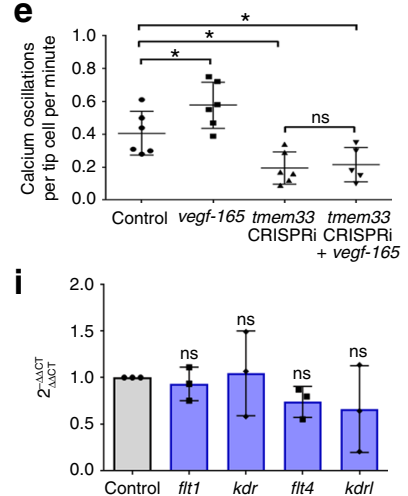
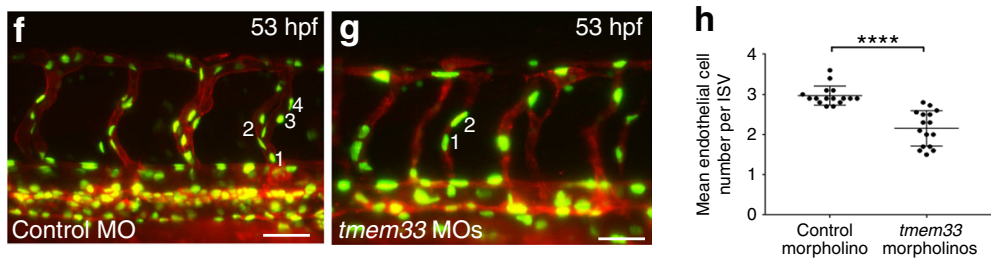

Fig. 4 tmem33 functions downstream of Vegfa during angiogenesis. a-e tmem33 knockdown prevents Vegfa-mediated increases in Ca2+ oscillations. Overexpression of vegfa $a_{165}$ significantly increases frequency of $\mathrm{Ca}^{2+}$ oscillations in SeAs, whereas tmem33 knockdown alone and in the presence of vegfa $a_{165}$ significantly reduce frequency of $\mathrm{Ca}^{2+}$ oscillations in SeAs. Intensity projection over time showing Ca ${ }^{2+}$ oscillations highlighting intensity change over duration of time lapse. Embryos display changes in fluorescence in SeA tip cells (white arrowheads) (two-way ANOVA with Holm-Sidak's corrections: ${ }^{\star} p=<0.05$. 2 Repeats, $n=3$ embryos per group) (scale bars $50 \mu \mathrm{m}$ ). f-h tmem33 knockdown using 0.4 ng morpholinos reduces EC number in ISVs at 53 hpf. $\mathbf{h}$ Unpaired $t$-test; ${ }^{\star \star \star \star} p<0.0001 ; t=6.507 ; \mathrm{df}=30 ; 2$ repeats; $n=8$ embryos per group. $\mathbf{i}$ VEGF receptor expression is not significantly affected in tmem33 crispants (one-way ANOVA with Dunnett's corrections; $p=<0.05, n=3$ repeats). Source data are provided as a Source Data file

Furthermore, $\operatorname{Tg}($ fli1a:dCas 9$)$ embryos injected with tmem33 sgRNA displayed absent parachordal lymphangioblasts (Fig. 3n, $\mathrm{o}$, red arrowheads) only in CFP-positive embryos, similar to tmem33 morphants (Fig. 11-o, red arrowheads). By contrast, $\mathrm{Tg}$ (fli1a:dCas9) embryos injected with tmem33 sgRNA displayed no significant difference in glomerular area between CFP-positive and -negative embryos (Fig. 3q-s, arrows). Collectively, these data demonstrate tissue-specific CRISPRi-mediated uncoupling of tmem 33 functions within the endothelium and pronephros, and indicate EC-specific and pronephros-specific functions of tmem33 during angiogenesis and pronephric development, respectively, in zebrafish. Importantly, all approaches to knockdown tmem33 function were compatible with normal gross embryonic morphology at phenotypic doses (Supplementary Fig. 11).

tmem33 functions downstream of VEGF during angiogenesis. Elevations in cytosolic $\mathrm{Ca}^{2+}$ are induced when EC tip cells respond to extracellular Vegfa via the $\mathrm{Kdrl}$ receptor $^{39}$ and EC $\mathrm{Ca}^{2+}$ oscillations are reduced in tip cells following tmem33 knockdown (Fig. 2a-e, Supplementary Movies 4, 5 and Supplementary Fig. 8d-h). We therefore sought to establish epistasis between VEGF signalling and tmem33. We overexpressed vegfa $a_{165}$ mRNA, which increases EC $\mathrm{Ca}^{2+}$ signalling (Supplementary Fig. 5g, h, j) and examined the effect on EC Ca ${ }^{2+}$ activity in the presence or absence of tmem33 CRISPRi knockdown (Fig. 4a-e and Supplementary Movies 6-9). As expected, vegfa $a_{165}$ overexpression increased the frequency of cytosolic $\mathrm{Ca}^{2+}$ oscillations in endothelial tip cells (Fig. 4a, b, arrowheads, e) but this was significantly reduced by simultaneous tmem 33 knockdown by CRISPRi (Fig. 4 d,e) similar to knockdown of tmem 33 alone without vegfa $a_{165}$ overexpression (Fig. 4c-e). Thus, tmem33 is required for Vegfa-induced $\mathrm{Ca}^{2+}$ oscillations in endothelial tip cells.

In addition, Vegfa promotes proliferation of intersegmental vessel (ISV) ECs via a process normally limited by Notch signalling 4 . We therefore asked whether tmem33 knockdown suppresses EC number in ISVs. Vessels are referred to as ISVs where the transgene employed did not allow distinction between SeAs and SeVs. Knockdown of tmem33 reduced EC number within ISVs (Fig. $4 \mathrm{f}-\mathrm{h}$ ), similar to previous studies following VEGF inhibition ${ }^{5}$. Furthermore, VEGF receptor expression was unaffected in tmem33 crispants (Fig. 4i). Taken together, these data demonstrate that tmem33 mediates the effects of VEGF during angiogenesis and is required for VEGF-mediated cytosolic $\mathrm{Ca}^{2+}$ signalling within endothelial tip cells. Furthermore, these data suggest tmem33 knockdown inhibits EC proliferation in response to VEGF.

TMEM33 is required for VEGFA-mediated angiogenesis in HUVECs. As tmem 33 mediates the effects of Vegfa in zebrafish, we next sought to establish whether this was conserved in humans. We used RNA interference to knock down TMEM33 in human umbilical vein ECs (HUVECs) (Fig. 5a) and examined the effect on EC migration and tube formation in response to VEGFA (Fig. 5b). Consistent with our findings in zebrafish, TMEM33deficient HUVECs migrated less than controls (Fig. 5b). In addition, HUVEC tubular morphogenesis induced by VEGFA was significantly reduced by TMEM33 knockdown (Fig. 5c), indicating the requirement for TMEM33 during the response to VEGFA is conserved in humans.

tmem33 knockdown reduces EC Notch and ERK signalling. VEGF signalling via VEGF receptor $2(k d r)$, and VEGFR4 $(k d r l)$ in zebrafish, induces transcription of $d l l 4$ in endothelial tip cells via the MEK-ERK signalling pathway. Upregulation of Dll4 in endothelial tip cells induces Notch signalling in neighbouring stalk cells, which balance tip and stalk cell identity via regulation of VEGFR expression ${ }^{7,41}$. As tmem33 mediates the response to VEGF, we examined whether Dll4/Notch signalling was 

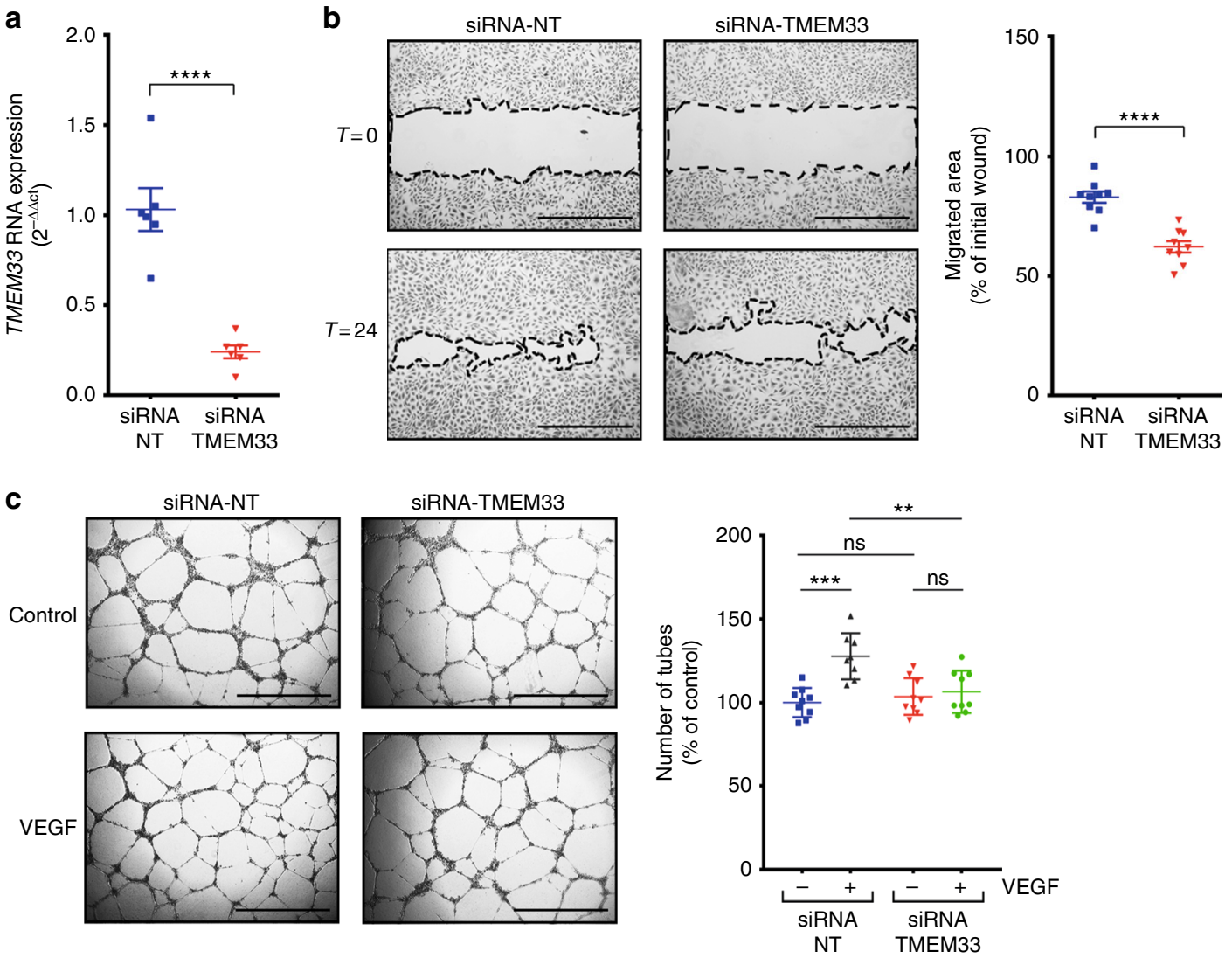

Fig. 5 TMEM33 is required for VEGFA-mediated angiogenesis in HUVECs. a TMEM33 expression is significantly reduced by small interfering RNA (siRNA) knockdown. $\mathbf{b}$ Representative images of wound-healing migration assays performed with HUVECs transfected with a non-targeting control siRNA (siRNANT) or a siRNA specific for human TMEM33 (siRNA-TMEM33) after incubation in culture medium supplemented with VEGF (25 ng/ml). Data are shown as mean $\pm \mathrm{SE}, n=9$, obtained from three independent experiments for comparison of cells transfected with siRNA-TMEM33 vs. siRNA-NT (unpaired twotailed, Student's $t$-test $\left.{ }^{* \star *} p \leq 0.001\right)$. Images were taken at time zero and after incubation for $24 \mathrm{~h}$. The migrated area was calculated by subtracting the value of the non-migrated area from the wound area at time zero and expressing this as a percentage of the total area at time zero. Scale bars, $1000 \mu \mathrm{m}$. c HUVECs transfected as described in a were plated on Growth-Factor Reduced Matrix (Geltrex) in Medium 200 containing 2\% FBS (Control), supplemented with VEGF $(25 \mathrm{ng} / \mathrm{ml})$ when indicated (VEGF). Images show representative fields from experiments quantified in the histogram. Data are shown as mean $\pm \mathrm{SE}, n=8$, obtained from three independent experiments; ns = nonsignificant; ${ }^{\star \star} p \leq 0.01$; ${ }^{\star \star *}$ one-way ANOVA with post-hoc Tukey's comparison test

$p \leq 0.005$. Scale bars, $1000 \mu \mathrm{m}$. Source data are provided as a Source Data file

perturbed by tmem33 knockdown. Using a transgenic Notch reporter $\operatorname{Tg}(\text { csl:venus })^{49}$, which reliably reports endothelial Notch signalling (Supplementary Fig. 12a-f), we observed no significant differences in reporter expression at $26 \mathrm{hpf}$ (Fig. 6a, b red arrowhead, c), but by $48 \mathrm{hpf}$, Notch reporter expression was significantly reduced in the DA (Fig. 6d, e, red arrowheads, f) and SeAs displayed reduced Venus fluorescence in tmem33 morphants (Fig. 6d, e, white arrowheads). In keeping with this, expression of dll4, notch $1 b$ and the Notch targets hey2/gridlock and her 12 were reduced by tmem 33 knockdown using global CRISPRi at $48 \mathrm{hpf}$ but not $26 \mathrm{hpf}$ (Fig. $6 \mathrm{~g}$, h and Supplementary Table 3). In situ hybridisation confirmed reduced arterial expression of all genes assayed by qPCR at $48 \mathrm{hpf}$ (Fig. 6i-p, arrowheads). Phosphorylation of the serine/threonine kinase ERK preferentially occurs in angiogenic ECs ${ }^{7}$. In keeping with this requirement downstream of VEGF signalling during angiogenesis, tmem33 knockdown reduced phosphorylated ERK (pERK) in sprouting SeAs (Fig. 6q-t, white arrowheads, u). pERK promotes expression of dll4 in zebrafish angiogenic $\mathrm{ECs}^{7,10}$ and dll4 expression was reduced by tmem 33 knockdown (Figs. 6h, n, arrowheads). These data indicate tmem33 is required for ERK phosphorylation and Notch signalling, which act downstream of VEGF in angiogenic ECs.
As tmem33 promotes sprouting angiogenesis cell autonomously (Fig. 3), we examined whether several other molecular and cellular consequences of tmem 33 inhibition were due to tmem33 function within ECs. Similar to knockdown of tmem33 by morpholino and CRISPRi, $\operatorname{Tg}($ flila:dCas 9 ) embryos injected with tmem33 sgRNA displayed reduced endothelial $\mathrm{Ca}^{2+}$ oscillations (Supplementary Fig. 13a-c, white arrowheads), reduced filopodia number and length (Supplementary Fig. 13d-g, black arrowheads), reduced EC number in ISVs (Supplementary Fig. 13h-j) and reduced endothelial Notch signalling (Supplementary Fig. 13k-m, arrowheads) in CFP-positive embryos in comparison with CFP-negative siblings. Collectively, this indicates tmem 33 is required cell autonomously to promote endothelial $\mathrm{Ca}^{2+}$ oscillations, filopodia formation, Notch signalling and to regulate EC number during angiogenesis.

SOCE inhibition inhibits angiogenesis. Although tmem 33 knockdown impairs angiogenesis and reduces endothelial cytosolic $\mathrm{Ca}^{2+}$ oscillations, it was possible that these effects were due to different roles of tmem 33 rather than causally linked. We therefore sought to determine whether inhibiting EC cytosolic $\mathrm{Ca}^{2+}$ release was sufficient to cause the observed phenotype of 

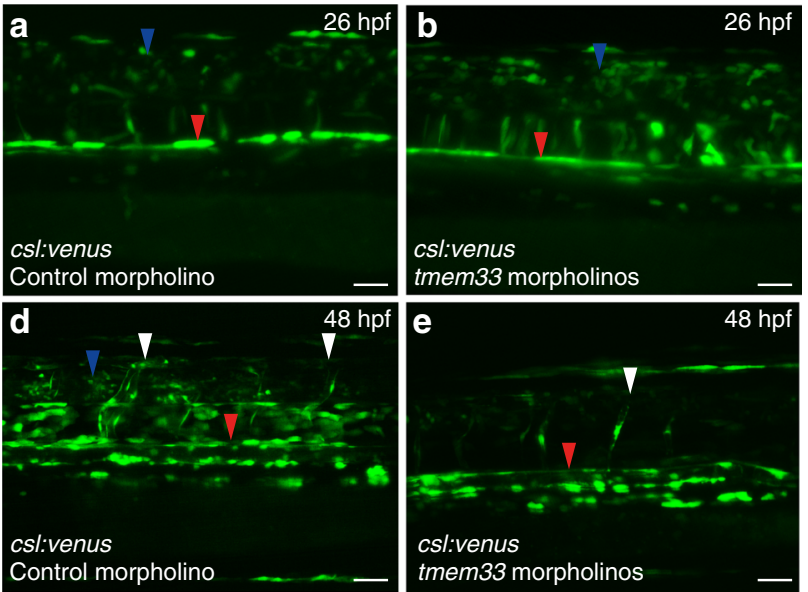

$\mathbf{g}$

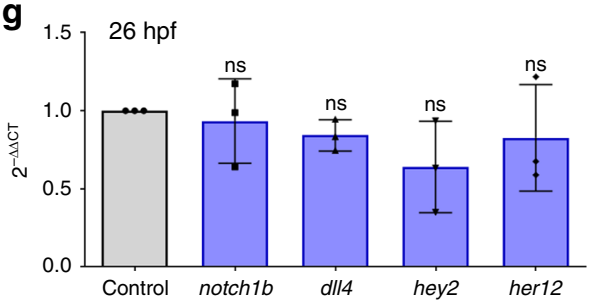

h

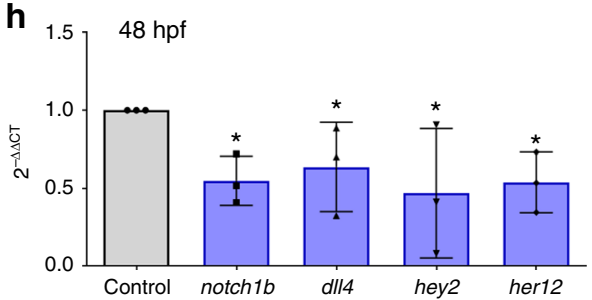

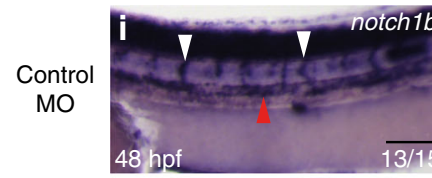
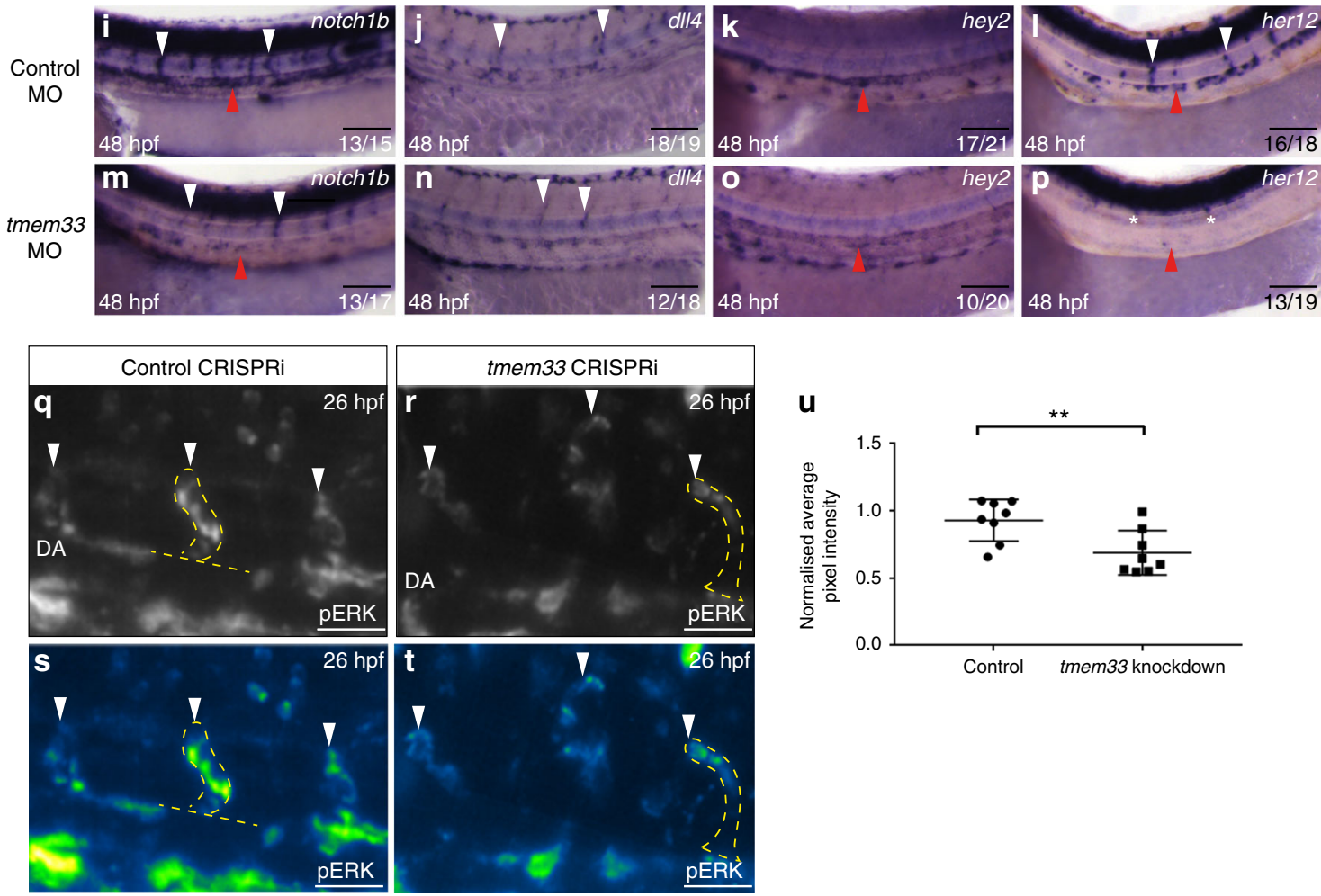

tmem33 knockdown. We inhibited SOCE using SKF-96365, a compound that inhibits STIM1 and TRPC1 function ${ }^{7,50}$. SKF96365 treatment between $21 \mathrm{hpf}$ and $27 \mathrm{hpf}$ reduced SeA length (Fig. $7 \mathrm{a}-\mathrm{c}$ arrowheads) as induced by tmem 33 knockdown (Fig. $1 \mathrm{j}, \mathrm{k}$ white arrowheads). Embryos treated from $21 \mathrm{hpf}$ until $50 \mathrm{hpf}$ displayed incomplete DLAV formation (Fig. $7 \mathrm{~d}-\mathrm{f}$, arrowheads) similar to tmem33 knockdown (Fig. 1n, o). Importantly, although tmem33 expression was unaffected by SKF-96365 treatment (Fig. $7 \mathrm{~g}$ ), frequency of endothelial $\mathrm{Ca}^{2+}$ oscillations was significantly reduced in tip cells (Fig. $7 \mathrm{~h}-\mathrm{j})$. Furthermore, Notch reporter expression (Fig. 7k-m) and endothelial tip cell filopodia number (Fig. $7 \mathrm{n}-\mathrm{p}$ ) was reduced in ECs following SKF96365 treatment similar to tmem33 knockdown (Fig. 6d-f and Fig. 2f-i, respectively). SKF-96365-treated embryos also displayed abnormal spade-shaped tip cell morphology (Fig. $7 \mathrm{~b}$, e, o, blue arrowheads) as induced by tmem 33 knockdown (Fig. 1k, white arrowhead). Given the similar angiogenic defects induced by tmem33 knockdown and SKF-96365 treatment, we examined whether inhibition of SOCE could account for reduced EC number and migration previously observed in tmem 33 morphants (Fig. 4f-h, and Supplementary Fig. 2). We treated embryos from $21 \mathrm{hpf}$ until $30 \mathrm{hpf}$ and quantified EC proliferation 
Fig. 6 tmem33 knockdown reduces endothelial Notch and ERK signalling during angiogenesis. a-c Notch reporter expression is unaffected by injection of $0.4 \mathrm{ng}$ tmem33 morpholinos at $26 \mathrm{hpf}$. Notch signalling activity in control embryos is present in neural tube (blue arrowhead) and DA (red arrowhead) (c, unpaired $t$-test; $t=0.5553 ; D F=20 ; 2$ repeats; $n=5$ or 6 embryos per group). Scale bars $50 \mu \mathrm{m}$. $\mathbf{d}$-f Injection of 0.4 ng tmem33 morpholinos reduces Notch reporter expression at $48 \mathrm{hpf}$. Notch expression in control $\mathrm{Tg}$ (csl:venus) embryos is present in the neural tube (blue arrowhead), DA (red arrowhead) and SeAs (white arrowheads) (f, unpaired $t$-test; $p=0.0451 ; t=2.138 ; \mathrm{DF}=20 ; 2$ repeats; $n=6$ and 4 embryos per group). Scale bars $50 \mu$ m. $\mathbf{g}$ Expression of notch1b, dll4, hey2 and her12 are not significantly altered by tmem33 CRISPRi at 26 hpf (one-way ANOVA using post hoc Tukey's comparison test. $n=3$ repeats). $\mathbf{h}$ Expression of notch $1 b, d l l 4$, hey 2 and her 12 are significantly reduced at 48 hpf by tmem33 CRISPRi (one-way ANOVA using post hoc Tukey's comparison test. ${ }^{\star} p=<0.05 ;{ }^{\star \star} p=<0.01 . n=3$ repeats). $\mathbf{i}-\mathbf{p}$ In comparison with control morphants (i-I), embryos injected with 0.4 ng tmem33 morpholino ( $\mathbf{m}-\mathbf{p})$ display reduced expression of notchib within the DA (i, $\mathbf{m}$, red arrowheads) and SeAs (i, $\mathbf{m}$, white arrowheads), dll4 within SeAs (j, $\mathbf{n}$, white arrowheads), hey2 within the DA (k, o, red arrowheads) and her12 within the DA (I, p, red arrowheads) and SeAs (I, p, white arrowheads). Scale bars $100 \mu \mathrm{m}$. q-t tmem33 knockdown by CRISPRi reduces endothelial ERK phosphorylation (q, $\mathbf{r}$, SeAs highlighted by yellow outline and white arrowheads). Intensity plot of ERK staining is shown ( $\mathbf{s}, \mathbf{t}$, SeAs highlighted by yellow outline and white arrowheads). Scale bars $50 \mu \mathrm{m}$. $\mathbf{u}$ tmem33 crispants display significantly reduced levels of pERK phosphorylation. Pixel intensity normalised to neural tube ERK fluorescence (unpaired $t$-test, ${ }^{\star \star} p=0.0097, t=2.993$ $D F=14 . n=2$ repeats, 4 embryos per group). Source data are provided as a Source Data file

and migration in the presence and absence of SKF-96365 between 24 and $30 \mathrm{hpf}$ (Supplementary Fig. 14 and Supplementary Movies 10, 11), and observed that both SeA migration (Supplementary Fig. 14a-e, arrowheads) and tip and stalk cell proliferation (Supplementary Fig. 14a-d,f-h) were significantly reduced in migrating SeAs treated with SKF-96365. Consistent with this, expression of VEGF receptors in tmem 33 crispants were unaffected (Fig. 4i) and thus unlikely to account for reduced EC number (Fig. 4f-h and Supplementary Fig. 13h-j). Collectively, these data demonstrate that SOCE inhibition impairs angiogenesis in vivo by limiting EC migration, proliferation, disrupting signalling pathways downstream of VEGF and inhibiting filopodia formation similar to tmem33 knockdown. This is consistent with our hypothesis that reduced cytosolic $\mathrm{EC} \mathrm{Ca}^{2+}$ oscillations account for the angiogenic defects observed caused by tmem33 knockdown. Thus, tmem33 is essential for VEGF-mediated endothelial $\mathrm{Ca}^{2+}$ oscillations that are required for tip cell filopodia formation, the downstream consequences of VEGF signalling and developmental angiogenesis (Fig. 8).

\section{Discussion}

No previous study has examined the function of tmem33 in a multicellular organism. Previous studies in yeast $\mathrm{t}^{20-22}$ and transformed human cells ${ }^{23,24}$ had localised tmem 33 to the ER, but no function has been demonstrated during development. We find tmem33 is expressed widely throughout the developing zebrafish embryo and localised to structures indicative of ER in zebrafish ECs. In keeping with its expression patterns, tmem 33 knockdown demonstrated a requirement for tmem33 during normal angiogenesis and pronephric development. Furthermore, TMEM33 is also required for VEGFA-mediated angiogenesis in human ECs, indicating a conserved function between zebrafish and human.

We find that tmem33 is required for Vegfa-mediated cytosolic $\mathrm{Ca}^{2+}$ oscillations in endothelial tip cells. Although increases in cytosolic $\mathrm{Ca}^{2+}$ are a well-established response to VEGF in $\mathrm{ECs}^{37}$, it remained unclear whether $\mathrm{Ca}^{2+}$ signalling was required for its effects in vivo and, if so, how $\mathrm{Ca}^{2+}$ co-ordinated EC behaviours during angiogenesis. Recent studies have established that Vegfa/ $\mathrm{Kdrl}$ signalling is required for endothelial $\mathrm{Ca}^{2+}$ oscillations during sprouting angiogenesis in vivo ${ }^{39}$. Our findings are consistent with these, as VEGFR inhibition abrogated $\mathrm{Ca}^{2+}$ oscillations and overexpression of vegf $a_{165}$ mRNA or Notch inhibition increased $\mathrm{Ca}^{2+}$ oscillations in tip cells. Reduction of $\mathrm{Ca}^{2+}$ oscillations in tip cells following either tmem 33 knockdown or acute SOCE inhibition were associated with reduced formation of filopodia, abnormal tip cell migration and aberrant anastomosis. Impaired SeA migration and DLAV anastomosis have been observed in embryos with reduced EC filopodia formation, suggesting this may contribute to the angiogenic defects induced by tmem33 knockdown ${ }^{42}$. Importantly, the frequency of cytosolic $\mathrm{Ca}^{2+}$ oscillations in tip cells was unaffected following loss of filopodia. This is in keeping with studies that demonstrated tip cells without filopodia retained their ability to respond to Vegfa ${ }^{42}$ and suggests reduced filopodia formation induced by tmem 33 knockdown was secondary to reduced EC $\mathrm{Ca}^{2+}$ oscillations. $\mathrm{Ca}^{2+}$ oscillations have been reported to promote $\mathrm{F}$-actin reorganisation and cell migration in vitro ${ }^{51,52}$. It therefore seems likely to be that tmem33-mediated cytosolic $\mathrm{Ca}^{2+}$ oscillations induced by VEGF signalling promote tip cell migration in vivo via organisation of the actin cytoskeleton. As such, tmem33-mediated $\mathrm{Ca}^{2+}$ oscillations permit tip cells to respond quickly to the proangiogenic Vegfa stimulus.

Vegfa-mediated activation of ERK signalling is also required for angiogenic sprouting and induction of tip cell marker expression including dll4 7,10,33,53. tmem33 knockdown reduced ERK phosphorylation and Dll4/Notch signalling, and delayed tip cell migration in a similar way to ERK inhibition ${ }^{7,53}$ without significantly affecting vegfr expression. This indicates tmem33 function contributes to activation of these pathways in vivo, likely to be via $\mathrm{Ca}^{2+}$-dependent mechanisms downstream of Vegfa. Interestingly, induction of Dll4/Notch signalling at $26 \mathrm{hpf}$ was not dependent on tmem33 function; however, its attenuation at 48 hpf, suggests tmem33 contributes to maintenance of Dll4/Notch signalling in ECs. Consistent with this, inhibition of SOCE reduced Notch reporter activity at $48 \mathrm{hpf}$, suggesting an input from VEGF-mediated calcium signalling between 1 and $2 \mathrm{dpf}$ to maintain Dll4/Notch signalling within developing arteries. Furthermore, recent studies indicate a requirement for $\mathrm{Ca}^{2+}$ binding during folding of Notch receptors and ligand engagement, including the Dll4-Notch 1 interaction ${ }^{54}$. Reduced expression of dll4 induced by tmem 33 knockdown is in keeping with observed reductions in ERK phosphorylation. ERK phosphorylation is required for development of the lymphatic system ${ }^{33-35,55}$; therefore, reduced ERK phosphorylation induced by tmem 33 knockdown may contribute to defective lymphatic sprouting.

Surprisingly, tmem 33 mutants exhibited normal angiogenesis, yet displayed substantial reductions in tmem 33 expression. The relative ease with which one can now generate targeted lesions within the zebrafish genome and widespread adoption of these approaches by the community has generated substantial evidence that many loss-of-function mutants fail to recapitulate morpholino-induced phenotypes ${ }^{56}$. Although this was initially attributed to well-established off-target effects of morpholinos ${ }^{57}$, recent studies have indicated a greater degree of compensation within the zebrafish genome than previously considered ${ }^{45}$. The tmem $33^{\text {sh } 443}$ allele is predicted to generate a premature stop codon within coding exon 4 , located 53 nucleotides downstream 


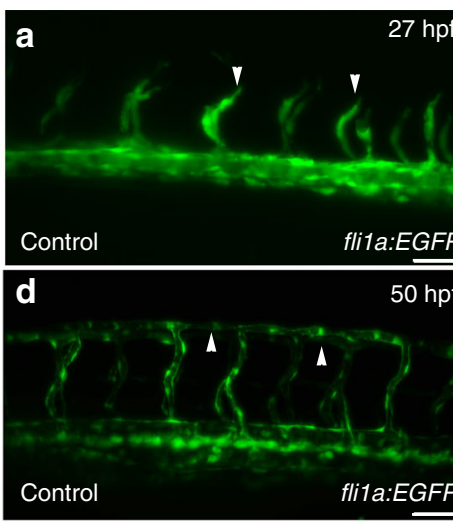

g
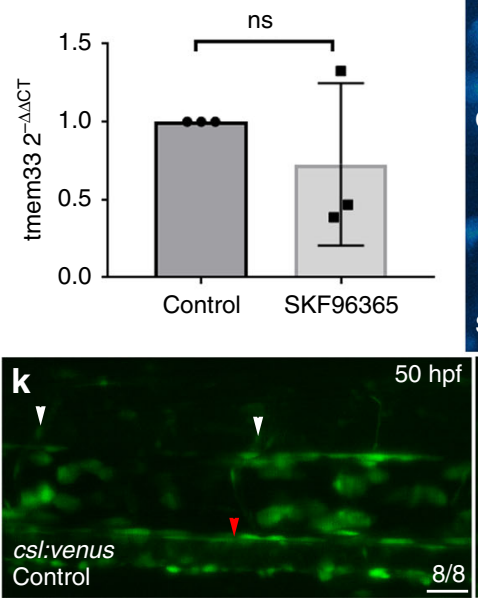
Contro

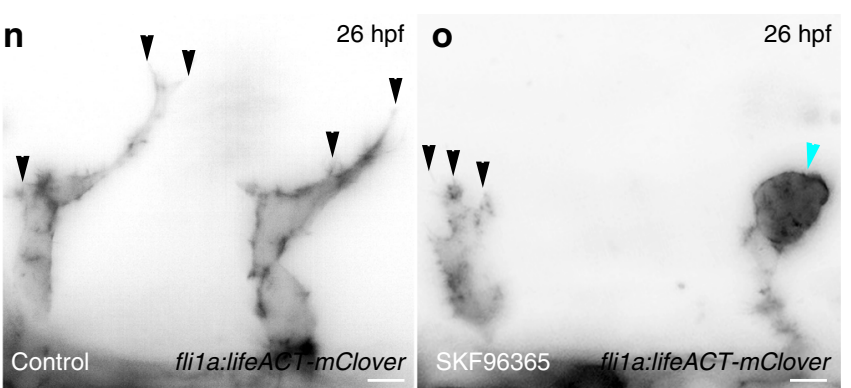

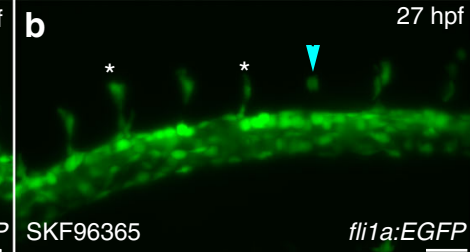
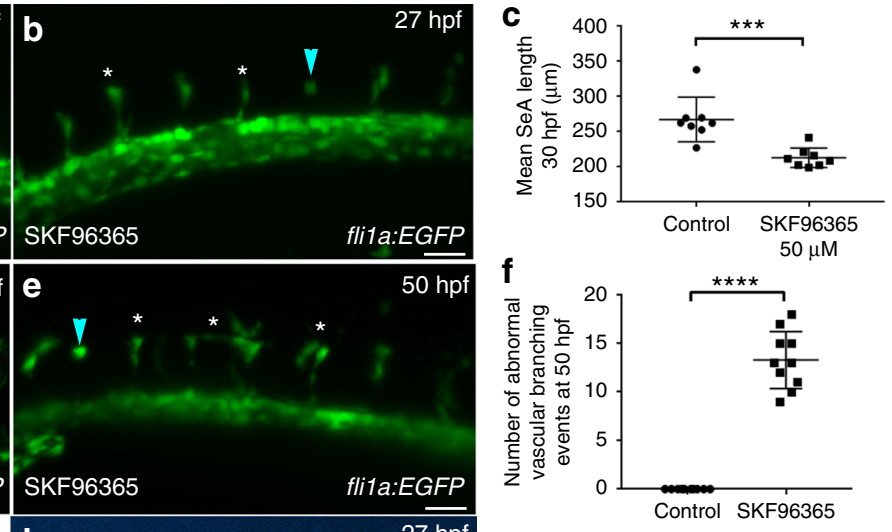

$27 \mathrm{hpf}$

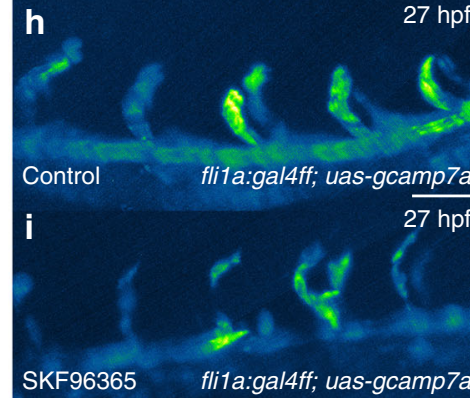

空畩

5

Control SKF96365
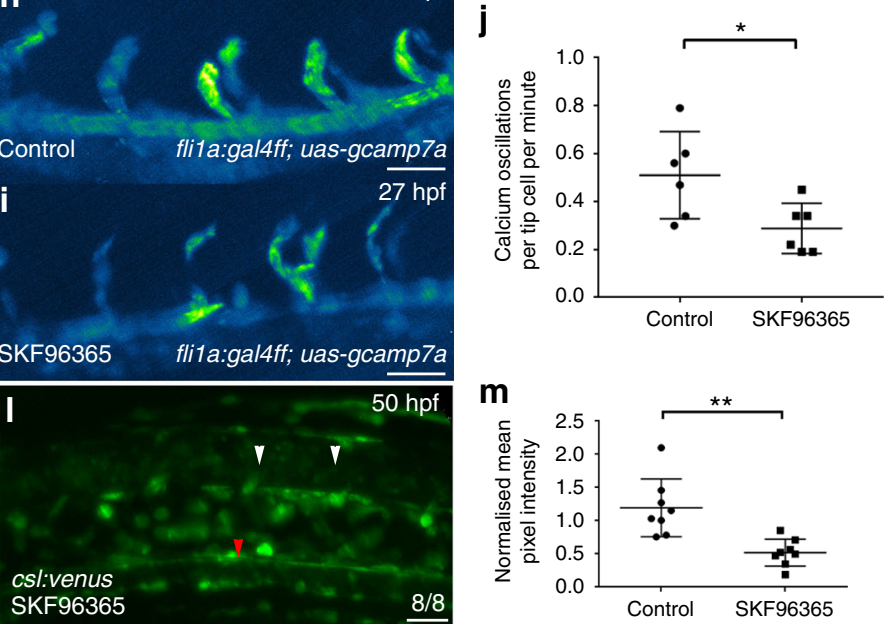

p

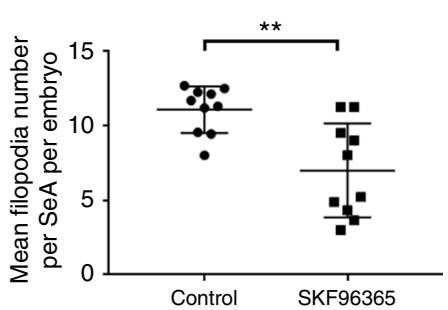

Fig. 7 SOCE inhibition limits sprouting angiogenesis and disrupts signalling pathways downstream of VEGF. a, b SKF9635 impairs angiogenesis in Tg(fli7a: EGFP) embryos (white arrowheads) and induces abnormal tip cell morphology (blue arrowhead) by 27 hpf. Scale bars $50 \mu$ m. c SKF-96365-treated embryos display reduced SeA length by $27 \mathrm{hpf}$ (unpaired $t$-test; $p=<0.0001 ; t=11.22 \mathrm{DF}=14 ; 2$ repeats, $n=4$ embryos per group). d, e SKF-96365 treatment impairs DLAV formation (white asterisks) in comparison with control embryos, which form the DLAV normally by 50 hpf (white arrowheads). Abnormal tip cell morphology (blue arrowhead) remain in SKF-96365-treated embryos by 50 hpf. Scale bars $50 \mu$ m. f SKF-96365-treated embryos display increased frequency of abnormal vascular branching (stunted SeAs or incomplete DLAV anastomosis) by 50 hpf (unpaired $t$-test; $p=<0.0001 ; t=14.28$ $\mathrm{DF}=18 . n=2$ repeats, $n=5$ embryos per group). $\mathbf{g}$ tmem33 expression is not significantly altered by SKF-96365 treatment (unpaired $t$-test; $t=0.911$, $\mathrm{DF}=4, n=3$ repeats). $\mathbf{h}$-j SKF-96365 reduces frequency of endothelial Ca ${ }^{2+}$ oscillations in Tg(fli7a:gal4FFubs3; uas-gcamp7a) embryos. Intensity projection over time showing calcium oscillations in control embryos, highlighting intensity change over duration of time lapse (unpaired $t$-test; $p=<0.05 ; t=2.594$, $\mathrm{DF}=10, n=2$ repeats, 3 embryos per group). $\mathbf{k}$, I Notch reporter expression is reduced in SeAs (white arrowheads) and DA (red arrowhead) of $T g$ (csl: venus) embryos. Scale bars $50 \mu \mathrm{m}$. $\mathbf{m}$ DA expression of Notch reporter is reduced by SKF treatment (unpaired $t$-test, ${ }^{* \star} p=0.0097, t=3.982 \mathrm{DF}=15,2$ repeats, $n=4$ embryos per group). $\mathbf{n}$, o SKF-96365 treatment reduces endothelial filopodia number (black arrowheads) and abnormal spade-shaped tip cell morphology (blue arrowhead), in Tg(flita:lifeact-mClover) embryos. Scale bars $5 \mu \mathrm{m}$. p SKF- 96365 treatment reduces mean filopodia number per SeA per embryo by $26 \mathrm{hpf}$ (unpaired $t$-test; $p=<0.05 ; t=2.525, \mathrm{DF}=10, n=2$ repeats, $n=5$ embryos per group). Source data are provided as a Source Data file

of an exon junction. This is consistent with the requirement for induction of nonsense-mediated decay ${ }^{58}$ and observed reductions in tmem33 expression. It is therefore likely that this allele represents a severe loss of function or null mutation, and that compensatory machinery exists within the zebrafish genome to account for normal angiogenesis in tmem $33^{\text {sh }} 443$ mutant embryos.

To circumvent these compensatory pathways, we have developed a strategy to transiently knock down gene function within 


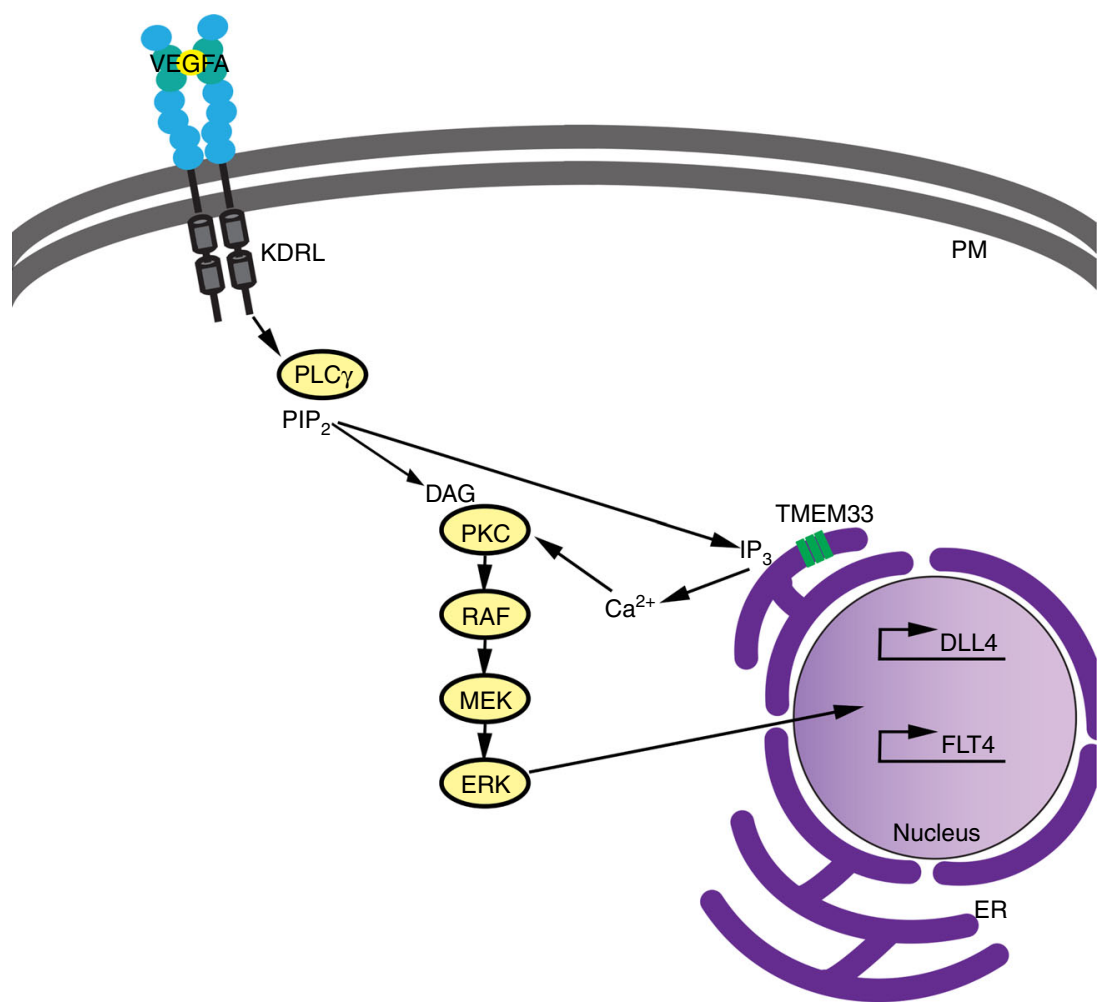

Fig. 8 Proposed model for tmem33 function in endothelial cells during angiogenesis. When VEGFA binds to its cognate receptor, e.g., Kdrl, the resulting phosphorylation of PLC $\gamma$ generates inositol 1,4,5-trisphosphate (IP3) and this, in turn, binds to IP3 receptors on the ER membrane, which release Ca2+ into the cytosol from intracellular stores. Our data suggest the VEGF-mediated release of $\mathrm{Ca}^{2+}$ from ER stores during angiogenesis is dependent on Tmem33 function within the ER membrane. Furthermore, resultant $\mathrm{Ca}^{2+}$ oscillations generated in tip cells downstream of Vegfa contribute to phosphorylation of ERK and induction or maintenance of downstream targets including DII4/Notch signalling, to co-ordinate cellular behaviours during vascular morphogenesis. ER, endoplasmic reticulum; $P$, plasma membrane

zebrafish endothelium and pronephros using tissue-specific CRISPRi. Using this approach we were able to uncouple tissuespecific functions of tmem 33 and show that tmem 33 is required cell autonomously in both tissues for their normal development. tmem33 functions within ECs to promote endothelial $\mathrm{Ca}^{2+}$ oscillations and filopodia formation during angiogenesis, and also to promote Notch signalling and regulate EC number. Tissuespecific CRISPRi offers a relatively straightforward method to study gene function within different tissues and represents an alternative to laborious cell transplantation procedures. Tissuespecific CRISPRi can be employed transiently to uncouple tissuespecific functions of genes, via co-injection of gene-targeting sgRNAs, dCas9 expression constructs and Tol2 mRNA. However, we observed more severe defects using this approach than when using stable transgenic lines expressing dCas9. We suspect this may be due to variable copy number integration mediated by Tol 2 and thus variable dCas9 expression, in addition to inherent toxicity of DNA microinjections. At minimal phenotypic doses, CRISPRi is less prone to induction of $p 53$ than some morpholinos and although this will vary depending upon the sgRNA, target and morpholino employed, our experience with the genes we have studied to date suggests CRISPRi may be susceptible to fewer off-target effects.

The localisation of tmem 33 within the ER in zebrafish ECs suggest it may have an important function in regulation of endothelial SOCE. Consistent with this, acute antagonism of SOCE induced similar reductions in cytosolic $\mathrm{Ca}^{2+}$ oscillations, filopodia formation and endothelial Notch activity as tmem33 knockdown, without affecting tmem33 expression. Furthermore, SOCE inhibition reduced EC proliferation, consistent with reduced EC number induced by tmem33 knockdown. Genome-wide protein interaction studies in Drosophila embryos, have shown that the Drosophila Tmem 33 orthologue, Kr-h2, physically interacts with SERCA ${ }^{59}$, and although this requires functional validation, the interaction appears to be conserved in humans. This suggests an ancestral function of Tmem33 in regulation of SOCE. Tmem 33 may also act to augment the activity of $\mathrm{Ca}^{2+}$ channels such as IP3R within the ER. Alternatively, tmem 33 may be involved in mediating cortical distribution of the ER, as has been described for a yeast orthologue Tts $1 \mathrm{p}^{22}$. Interestingly, rapid changes in cytosolic $\mathrm{Ca}^{2+}$ are known to induce dynamic changes in actin cytoskeleton organisation in vitro ${ }^{60}$. The actin cytoskeleton has been linked to normal SOCE function by facilitating interaction of ER-resident proteins with their plasma membrane counterparts ${ }^{19,61}$. As tmem 33 knockdown reduces $\mathrm{Ca}^{2+}$ oscillations and inhibits filopodia formation, this represents an intriguing possibility. VEGF has been shown to induce distinct modalities of calcium oscillations that correlate with different EC behaviours, including migration and proliferation ${ }^{62}$. We find that inhibition of SOCE impairs both migration and proliferation of ECs and knockdown of tmem33 similarly impairs EC migration and reduces EC number, suggesting an essential requirement for $\mathrm{Ca}^{2}$ ${ }^{+}$oscillations in both processes. The endothelial actin cytoskeleton is necessary for efficient mitosis and migration, and is notably disrupted by tmem33 knockdown or SOCE inhibition. This suggests that reduced EC proliferation and migration induced by inhibition of SOCE or reduced migration and EC number induced by tmem33 knockdown may represent a consequence of $\mathrm{Ca}^{2+}$-dependent disruption of the endothelial actin cytoskeleton. Further studies will be required to address the precise function of Tmem33-mediated $\mathrm{Ca}^{2+}$ within the ER in ECs in response to VEGF. 


\section{Methods}

Zebrafish strains, morpholinos and sgRNA. All zebrafish were maintained according to institutional and national ethical and animal welfare guidelines. All experiments were performed under UK Home Office licences 40/3708 and 70/8588. The following published zebrafish lines were employed: $T g(f l i 1 a: E G F P)^{y 1} 63 \mathrm{Tg}$ $(-0.8 f l t 1: e n h R F P)^{h u 533325}, \mathrm{Tg}(-26 w t 1 b: E G F P)^{l i 1}$ 64, $\mathrm{Tg}(\mathrm{flk1} \text { :EGFP-NLS) })^{z f 109} 65, \mathrm{Tg}$ (kdrl:HRAS-mCherry-CAAX) $)^{\text {s916 } 66}$ and $T g(c s l: v e n u s)^{9 m c 6149}$. For details on mutant and transgenic line generation, see Supplementary Methods. A quantity of 0.2 or 2 $\mathrm{ng}$ of Sp2b and Sp3 morpholinos were injected. Where these were co-injected, a total of $0.4 \mathrm{ng}$ or $4 \mathrm{ng}$ was injected. Four nanograms of morpholino was found to exhibit some induction of p53 (Supplementary Fig. $7 \mathrm{~h}$ ) and was titrated to $0.4 \mathrm{ng}$ to reduce this; however, phenotypes were equivalent at each dose. One nanogram of each sgRNA was injected alongside $500 \mathrm{pg}$ of $d \operatorname{Cas} 9 \mathrm{mRNA}^{45}$. Negative control sgRNAs contained the DNA-binding element of the active gRNA but lacked the stem-loop forming region associated with binding to the Cas9 or dCas9 protein For morpholino, sgRNA and primer sequences, see Supplementary Methods.

\section{Generation of tmem33 $3^{\text {sh }} 443$ mutant allele. TALENs specific for tmem 33} (ENSDARG00000041332) were designed against the following sequence 5'cttcctggcccaggctt- $3^{\prime}$ targeting an EcoNI site within exon 3. TALENs were assembled using the Golden Gate TALEN and TAL Effector Kit (Addgene, MA, USA) ${ }^{44}$ to generate the pTmem33Tal1L\&R plasmids. Following linearisation of pTmem33Tal1L\&R with NotI, capped mRNA was generated by in vitro transcription and 1500 pg TALEN mRNA was injected per embryo. Individual G0 embryos were tested by PCR and restriction fragment length polymorphism using tmem33ex3 F and tmem33ex3R primers (Table S1), to identify somatic mutations that destroyed the EcoNI restriction site. The progeny of TALEN-injected G0 adults were incrossed and genotyped to confirm the presence of the sh443 allele. All studies were performed using F3 and F4 generations.

Generation of fli1a:dCas 9 and enpep:dCas 9 constructs. pME-dCas 9 was generated by cloning the $n l s-d \operatorname{Cas} 9-n l s$ coding sequence from pT3TS-dCas9, a kind gift of Didier Stainier, into pME-MCS2, which contained the multiple cloning site of pCS2+. flila:dCas9;cryaa:cfp and enpep:dCas9; cryaa:cfp constructs (Supplementary Fig. 10a) were subsequently generated using the Tol2 Kit via standard methods ${ }^{67}$. Each construct was injected into Nacre embryos alongside Tol2 mRNA at $25 \mathrm{pg} / \mathrm{nl}$.

Generation of transgenic lines. $\operatorname{Tg}\left(f l i 1 a: G a l 4 F F^{u b s 3} ; U A S-G C a M P 7 a\right)^{s h 392}$ was generated via injection of pTol2UASGCaMP7a into progeny of $\operatorname{tg}(f l i 1 a: G a l 4 F F)$ ubs 368 heterozygous outcross alongside tol2 mRNA according to standard proto$\operatorname{cols}^{67}$. Tg(flila:LifeAct- $m$ Clover) ${ }^{\text {sh } 467}$ was generated by fusing the LifeAct F-Actin binding motif ${ }^{69}$ to the $\mathrm{N}$ terminus of mClover by PCR (Table S1) to generate pMELifeAct-mClover. flila:LifeAct-mClover construct was generated using the tol2 Kit via standard methods ${ }^{67}$ and the following components: flila enhancer/promoter ${ }^{70}$, pME-LifeAct-mClover, pDestTol2-pA2 ${ }^{67}$, and p3E-SV40pA ${ }^{67} . \mathrm{Tg}($ flila:DsRedEx2) sh 495 was generated using the tol2 Kit via standard methods ${ }^{67}$ and the following components: flila enhancer/promoter ${ }^{70}$, pENTRDSRedEx $2^{70}$, pDestTol2-pA2 $2^{67}$, and $\mathrm{p} 3 \mathrm{E}-\mathrm{SV} 40 \mathrm{pA}^{67}$. $\mathrm{Tg}(f l i 1 a: A C-T a g R F P)^{\text {sh511 }}$ was generated by amplifying the actin- $\mathrm{V}_{\mathrm{H}} \mathrm{H}$-TagRFP coding sequence from pAC-TagRFP (Chromotek) and adding attB1/B2R sites (Table S1) to generate pME-AC-TagRFP. flila:AC-tagRFP construct was generated using the Tol $2 \mathrm{Kit}^{67}$ and components listed above. $\mathrm{Tg}$ (flila: $d$ Cas 9 , cryaa:Cerulean) $)^{\text {sh } 512}$ was generated by co-injecting fli1a:dCas9, cryaa:cfp plasmid with Tol2 mRNA. Embryos were injected at one-cell stage with $25 \mathrm{ng} / \mu \mathrm{l}$ Tol2 mRNA and corresponding plasmid DNA.

mRNA microinjections. Microinjections of both $v e g f a_{165}{ }^{63}$ (300 pg), tmem33 (250 $\mathrm{pg})$ and tmem33-gfp (1200 pg) mRNA were performed on single-cell-stage embryos with $1 \mathrm{nl}$ injection volume.

RNA in situ hybridisation and immunohistochemistry. Alkaline phosphatase wholemount in situ hybridisation experiments were performed using standard methods as described previously ${ }^{71}$. Detailed protocols are available upon request. EC $d$ Cas 9 expression was detected using Cas9 in situ probe $\mathrm{p}^{72}$. Immunohistochemistry to detect pERK was performed using Phospho-p44/42 Erk1.2 (Thr202. Tyr204) Rabbit mAb (\#4370, cell signal, 1:250) and quantified by normalising EC signal against ERK staining within the neural tube as described ${ }^{7,73}$. Immunohistochemistry to detect dCas9 was performed on whole-mount and sectioned embryos as described ${ }^{74}$ using mouse anti-CRISPR/Cas9 (7A9-3A3) (Novus Biologicals, NBP2-36440, 1:100), chicken anti-GFP (Abcam, ab13970, 1:500) primary antibodies, goat anti-mouse IgG (H\&L) Alexa Fluor ${ }^{\circledR} 647$ (Thermo Fisher A21235, 1:1000), and goat anti-chicken IgY (H\&L) Alexa Fluor ${ }^{\circledR} 488$ (Thermo Fisher A11039, 1:1000) secondary antibodies. Sectioned embryos were fixed at $3 \mathrm{dpf}$ in $4 \%$ paraformaldehyde overnight, washed into $30 \%$ sucrose and sectioned at $14 \mu \mathrm{m}$ thickness on a Jung Frigocut cryostat (Leica).

Quantitative RT-PCR. qRT-PCR was performed using Taqman ${ }^{\text {tx }}$ assays according to the manufacturer's instructions (see Supplementary Methods for probe details). RNA was extracted from batches of 20 or 30 whole embryos per repeat using
Trizol $^{\oplus}$, as a template for complementary DNA synthesis (Verso ${ }^{\mathrm{mt}} \mathrm{cDNA}$ synthesis kit, Thermo Fisher). A 7900 Real-Time PCR system (Applied Biosystems) was used for $\mathrm{qPCR}$ experiments. Gene expression levels were normalised to eef1 $\alpha$ and for each comparison the experimental group was normalised to their relative control unless otherwise stated. All experiments were performed in triplicate. Results display triplicate $2^{-\Delta \Delta C T}$ values and SEM (unless otherwise stated).

Image acquisition and analysis. For light sheet imaging, embryos were anaesthetised using tricaine in E3 medium and mounted in 1\% agarose. A light sheet Z.1 system was used and images were acquired using ZEN software (Zeiss). For confocal microscopy, still images were taken using an Ultraview VOX confocal spinning disc system (Perkin Elmer), Zeiss LSM880 with Airyscan and Leica SP5. Images were analysed using Volocity ${ }^{\oplus}$ V.3.2, ZEN software (Zeiss) and Leica LCS Confocal software. In cases where samples displayed drift during time lapses, these were corrected using the translation algorithm in ZEN (Blue Edition).

Calcium imaging. Calcium oscillations were quantified using 12-14 SeAs between 24 and $28 \mathrm{hpf}$ on a Zeiss Lightsheet Z.1. All time lapses were taken at $3 \mu \mathrm{m} z$ intervals at 20 frames per second (fps) with an acquisition time of $4-6 \mathrm{~s}$ per stack using a $\times 20$ objective lens for $300 \mathrm{~s}$. Changes in fluorescence over time were measured throughout the DA to establish a baseline. Changes in fluorescence measured in tip cells were determined to be peaks by subtracting mean DA fluorescence. Changes in fluorescence were normalised to DA baseline values. In experiments where ectopic $\mathrm{Ca}^{2+}$ oscillations were induced in the DA, the mean DA value of control embryos was used to normalise datasets. Absolute calcium peak number was quantified and averaged per SeA, per minute. FIJI was employed to quantify fluorescence intensity. Tip cells were manually segmented as regions of interest and mean and peak fluorescence (arbitrary units) were determined.

Pharmacological treatment. VEGF inhibition using Tivozanib/AV951 (500 nM) Notch inhibition using DAPT $(100 \mu \mathrm{M})$ or inhibition of F-actin polymerisation using Latrunculin B $(380 \mathrm{nM})$ was conducted using dechorionated embryos between 24 and $28 \mathrm{hpf}$ in E3 medium with dimethyl sulfoxide (DMSO) as a control. SERCA inhibition using Thapsigargin $(5 \mu \mathrm{M})$ was performed for $45 \mathrm{~min}$ before immediate imaging using a Perkin Elmer Spinning Disk. SOCE inhibition using SKF-963656 $(50 \mu \mathrm{M})$ dissolved in water was performed on dechorionated embryos using untreated embryos as control. The effect of SKF-96365 treatment on EC proliferation and migration (Supplementary Fig. 14) was performed using continuous exposure to compound within a light sheet chamber using SKF-96365 at a concentration of $50 \mu \mathrm{M}$ within $0.7 \%$ agarose mounting medium and surrounding E3 medium + Tricaine. Embryos were treated from $21 \mathrm{hpf}$ and imaged between 24 and $30 \mathrm{hpf}$. One to two embryos were imaged for each individual condition and three congruent repeats of each condition were performed.

Calculation of DA blood flow velocimetry. Single $Z$-plane time lapses of $3 \mathrm{dpf}$ zebrafish DA were acquired at $66 \mathrm{fps}$ using a Zeiss Lightsheet Z.1 microscope. The resulting images were subjected to axial line scan particle image velocimetry designed for Lightsheet imaging using custom authored scripts in MATLAB $2017 \mathrm{~b}^{\oplus}$. Each time lapse was analysed frame by frame using intensity-based thresholding of circulating erythrocytes. Thresholded images were made binary followed by conversion of two-dimensional image to one-dimensional (1D) signal by summing up in ' $y$ ' direction (all column pixels). The 1D signal was low pass filtered to remove spurious and highly correlated pixels (low correlation implies displacement of $1 \mathrm{D}$ wave signal). Cross-correlation across time was calculated on filtered images for each time frame using inbuilt MATLAB $2017 \mathrm{~b}^{\circledR}$ functions. Thus, the obtained correlation (in pixels/frames) was then converted to $\mathrm{mm} / \mathrm{s}$ using the following equation:

$$
\operatorname{Velocity}(\mathrm{mm} / \mathrm{s})=\frac{\text { Correlation }(\text { pixels } / \text { frame }) \times \mathrm{fps} \times 10^{-3}}{\text { Scale }(\text { pixels } / \mu \mathrm{m})}
$$

Algorithm is available on request.

siRNA-TMEM33 knockdown in HUVEC. Small interfering RNA (siRNA)-mediated knockdown of TMEM33 gene expression in HUVEC cells was performed using ON-TARGET-plus SMART pool human TMEM33 (Thermo Scientific). ONTARGET-plus non-targeting pool control duplexes (Thermo Scientific) were used as a control. For siRNA transfection, HUVECs (Cellworks, ZHC-2301) were plated in $0.1 \%$ gelatin pre-coated six-well tissue culture plates $\left(3 \times 10^{5}\right.$ cells/well $)$ and incubated overnight. The following morning, cells were washed with phosphatebuffered saline and incubated in serum-free, antibiotic-free OPTIMEM medium for $1 \mathrm{~h}$. Then, $100 \mathrm{pmol}$ of siRNA/well were transfected using $5 \mu \mathrm{l}$ of Lipofectamine 2000 (Thermo Scientific). Transfection medium was removed after incubation for $6 \mathrm{~h}$ and substituted by ECGM supplemented with ECGM-supplement mix and 1\% penicillin/streptomycin/amphotericin B (Sigma-Aldrich). Cells were incubated for $72 \mathrm{~h}$ and then used for further experiments. 
Wound-healing EC migration assay. Cell migration of HUVECs and MLECs, treated as indicated, was assayed using the Cytoselect ${ }^{\text {ow }}$ 24-well Wound Healing Assay kit (Cell Biolabs). Images were captured with a Nikon DSFil digital camera coupled to a Nikon ECLIPSE TS100 microscope at $\times 4$ magnification. Cell-free area was quantified with ImageJ software.

Matrigel EC tube-formation assay. HUVECs transfected with the corresponding siRNA were plated onto Geltrex Reduced Growth-Factor Matrix (Invitrogen) in Medium 200 with no phenol red and supplemented with $2 \%$ foetal calf serum and VEGF $(25 \mathrm{ng} / \mathrm{ml})$ when indicated. Tube formation was quantified after incubation at $37^{\circ} \mathrm{C}$ for $16 \mathrm{~h}$. Images were recorded with a Nikon DSFil digital camera coupled to a Nikon ECLIPSE TS100 microscope at $4 \times$ magnification.

Statistical analysis. All statistical analysis employed two-tailed tests and are described in figure legends. All error bars display the mean and SD, except for qPCR error bars, which display the mean and SEM. Numbers of experimental repeats are listed in figure legends. $P$-values, unless exact value is listed, are as follows: ${ }^{*}<0.05,{ }^{* *}<0.01,{ }^{* *}<0.001$ and ${ }^{* * *}<0.0001$. F-values, $t$-values and degrees of freedom are listed in individual figure legends.

Reporting Summary. Further information on experimental design is available in the Nature Research Reporting Summary linked to this Article.

\section{Data availability}

The datasets generated during and/or analysed during the current study are available from the corresponding author on reasonable request. The source data underlying Figs. 1l-m, 2b, d, e, h, i, 3g, j, m, p, 4e, h, i, 5a-c, 6c, f-h, u and 7c, f, g, j, m, p, and Supplementary Figs. 2e, 3d, 4d, e, 5b, d, f, h-j, 6c, f, 7e, h, l, o, 8c, e, g, h, k-m, 9j, k, 10k, $\mathrm{l}, 12 \mathrm{c}, \mathrm{f}, 13 \mathrm{c}, \mathrm{f}, \mathrm{g}, \mathrm{j}, \mathrm{m}$ and $14 \mathrm{e}-\mathrm{h}$ are provided as a Source Data file. A Reporting Summary for this Article is available as a Supplementary Information file.

Received: 15 August 2017 Accepted: 21 January 2019

Published online: 13 February 2019

\section{References}

1. Potente, M., Gerhardt, H. \& Carmeliet, P. Basic and therapeutic aspects of angiogenesis. Cell 146, 873-887 (2011)

2. Koch, S., Tugues, S., Li, X., Gualandi, L. \& Claesson-Welsh, L. Signal transduction by vascular endothelial growth factor receptors. Biochem. J. 437, 169-183 (2011).

3. Gerhardt, H. et al. VEGF guides angiogenic sprouting utilizing endothelial tip cell filopodia. J. Cell Biol. 161, 1163-1177 (2003).

4. Siekmann, A. F. \& Lawson, N. D. Notch signalling limits angiogenic cell behaviour in developing zebrafish arteries. Nature 445, 781-784 (2007)

5. Costa, G. et al. Asymmetric division coordinates collective cell migration in angiogenesis. Nat. Cell Biol. 18, 1292-1301 (2016).

6. Meadows, K. N., Bryant, P. \& Pumiglia, K. Vascular endothelial growth factor induction of the angiogenic phenotype requires Ras activation. J. Biol. Chem. 276, 49289-49298 (2001).

7. Shin, M. et al. Vegfa signals through ERK to promote angiogenesis, but not artery differentiation. Development 143, 3796-3805 (2016).

8. Takahashi, T., Yamaguchi, S., Chida, K. \& Shibuya, M. A single autophosphorylation site on KDR Flk-1 is essential for VEGF-A-dependent activation of PLC-g and DNA. EMBO J. 20, 2678-2778 (2001).

9. Tran, Q. K. \& Watanabe, H. Calcium signalling in the endothelium. Handb. Exp. Pharmacol. 145-187 (2006).

10. Wythe, J. D. et al. ETS factors regulate Vegf-dependent arterial specification. Dev. Cell. 26, 45-58 (2013).

11. Blanco, R. \& Gerhardt, H. VEGF and Notch in tip and stalk cell selection. Cold Spring Harb. Perspect. Med. 3, a006569 (2013).

12. Parekh, A. B. Decoding cytosolic Ca2+ oscillations. Trends Biochem. Sci. $\mathbf{3 6}$, 78-87 (2011)

13. Oike, M., Gericke, M., Droogmans, G. \& Nilius, B. Calcium entry activated by store depletion in human umbilical vein endothelial cells. Cell Calcium 16, 367-376 (1994).

14. Vaca, L. \& Kunze, D. L. Depletion of intracellular Ca2 stores activates a $\mathrm{Ca}(2)-$ selective channel in vascular endothelium. Am. J. Physiol. 267, C920-C925 (1994).

15. Cheng, K. T., Liu, X., Ong, H. L., Swaim, W. \& Ambudkar, I. S. Local Ca(2) +entry via Orail regulates plasma membrane recruitment of TRPC1 and controls cytosolic $\mathrm{Ca}(2)+$ signals required for specific cell functions. PLoS Biol. 9, e1001025 (2011).

16. Feske, S. et al. A mutation in Orail causes immune deficiency by abrogating CRAC channel function. Nature 441, 179-185 (2006).
17. Liou, J. et al. STIM is a Ca2+sensor essential for $\mathrm{Ca} 2+-$ store-depletiontriggered Ca2+ influx. Curr. Biol. 15, 1235-1241 (2005).

18. Roos, J. et al. STIM1, an essential and conserved component of store-operated Ca2+ channel function. J. Cell Biol. 169, 435-445 (2005).

19. van Vliet, A. R. et al. The ER stress sensor PERK coordinates ER-plasma membrane contact site formation through interaction with Filamin-A and Factin remodeling. Mol. Cell 65, 885-899 (2017)

20. Floch, A. G. et al. Nuclear pore targeting of the yeast Pom33 nucleoporin depends on karyopherin and lipid binding. J. Cell Sci. 128, 305-316 (2015).

21. Chadrin, A. et al. Pom33, a novel transmembrane nucleoporin required for proper nuclear pore complex distribution. J. Cell Biol. 189, 795-811 (2010).

22. Zhang, D., Vjestica, A. \& Oliferenko, S. The cortical ER network limits the permissive zone for actomyosin ring assembly. Curr. Biol. 20, 1029-1034 (2010).

23. Sakabe, I., Hu, R., Jin, L., Clarke, R. \& Kasid, U. N. TMEM33: a new stressinducible endoplasmic reticulum transmembrane protein and modulator of the unfolded protein response signaling. Breast Cancer Res. Treat. 153, 285-297 (2015).

24. Urade, T., Yamamoto, Y., Zhang, X., Ku, Y. \& Sakisaka, T. Identification and characterization of TMEM33 as a reticulon-binding protein. Kobe J. Med. Sci. 60, E57-E65 (2014).

25. Bussmann, J. et al. Arteries provide essential guidance cues for lymphatic endothelial cells in the zebrafish trunk. Development 137, 2653-2657 (2010).

26. Yaniv, K. et al. Live imaging of lymphatic development in the zebrafish. Nat. Med. 12, 711-716 (2006).

27. Bahary, N. et al. Duplicate VegfA genes and orthologues of the KDR receptor tyrosine kinase family mediate vascular development in the zebrafish. Blood 110, 3627-3636 (2007).

28. Covassin, L. D., Villefranc, J. A., Kacergis, M. C., Weinstein, B. M. \& Lawson, N. D. Distinct genetic interactions between multiple Vegf receptors are required for development of different blood vessel types in zebrafish. Proc. Natl Acad. Sci. USA 103, 6554-6559 (2006).

29. Lawson, N. D., Mugford, J. W., Diamond, B. A. \& Weinstein, B. M. phospholipase C gamma-1 is require downstream of vascular endothelial growth factor during arterial development. Genes Dev. 17, 1346-1351 (2003).

30. Nasevicius, A., Larson, J. \& Ekker, S. C. Distinct requirements for zebrafish angiogenesis revealed by a VEGF-A morphant. Yeast 17, 294-301 (2000).

31. Rossi, A. et al. Regulation of Vegf signaling by natural and synthetic ligands. Blood 128, 2359-2366 (2016)

32. Astin, J. W. et al. Vegfd can compensate for loss of Vegfc in zebrafish facial lymphatic sprouting. Development 141, 2680-2690 (2014).

33. Shin, M. et al. Vegfc acts through ERK to induce sprouting and differentiation of trunk lymphatic progenitors. Development 143, 3785-3795 (2016).

34. Hogan, B. M. et al. Vegfc/Flt4 signalling is suppressed by Dll4 in developing zebrafish intersegmental arteries. Development 136, 4001-4009 (2009).

35. Le Guen, L. et al. Ccbe1 regulates Vegfc-mediated induction of Vegfr3 signaling during embryonic lymphangiogenesis. Development 141, 1239-1249 (2014)

36. Villefranc, J. A. et al. A truncation allele in vascular endothelial growth factor $c$ reveals distinct modes of signaling during lymphatic and vascular development. Development 140, 1497-1506 (2013).

37. Brock, T. A., Dvorak, H. F. \& Senger, D. R. Tumor-secreted vascular permeability factor increases cytosolic $\mathrm{Ca} 2+$ and von Willebrand factor release in human endothelial cells. Am. J. Pathol. 138, 213-221 (1991)

38. Zhou, Y. et al. STIM1 gates the store-operated calcium channel ORAI1 in vitro. Nat. Struct. Mol. Biol. 17, 112-116 (2010).

39. Yokota, Y. et al. Endothelial Ca 2+ oscillations reflect VEGFR signalingregulated angiogenic capacity in vivo. eLife 4, e08817 (2015).

40. Lytton, J., Westlin, M. \& Hanley, M. R. Thapsigargin inhibits the sarcoplasmic or endoplasmic reticulum Ca-ATPase family of calcium pumps. ournal Biol. Chem. 266, 17067-17071 (1991).

41. Phng, L. K. \& Gerhardt, H. Angiogenesis: a team effort coordinated by notch Dev. Cell. 16, 196-208 (2009).

42. Phng, L. K., Stanchi, F. \& Gerhardt, H. Filopodia are dispensable for endothelial tip cell guidance. Development 140, 4031-4040 (2013).

43. Tammela, T. et al. Blocking VEGFR-3 suppresses angiogenic sprouting and vascular network formation. Nature 454, 656-660 (2008).

44. Cermak, T. et al. Efficient design and assembly of custom TALEN and other TAL effector-based constructs for DNA targeting. Nucleic Acids Res. 39, e82 (2011).

45. Rossi, A. et al. Genetic compensation induced by deleterious mutations but not gene knockdowns. Nature 524, 230-233 (2015).

46. El-Brolosy, M. A. \& Stainier, D. Y. R. Genetic compensation: a phenomenon in search of mechanisms. PLoS Genet. 13, e1006780 (2017).

47. Larson, M. H. et al. CRISPR interference (CRISPRi) for sequence-specific control of gene expression. Nat. Protoc. 8, 2180-2196 (2013).

48. Leslie, J. D. et al. Endothelial signalling by the Notch ligand Delta-like 4 restricts angiogenesis. Development 134, 839-844 (2007). 
49. Gray, C. et al. Loss of function of parathyroid hormone receptor 1 induces Notch-dependent aortic defects during zebrafish vascular development. Arterioscler. Thromb. Vasc. Biol. 33, 1257-1263 (2013).

50. Zou, J. J., Gao, Y. D., Geng, S. \& Yang, J. Role of STIM1/Orail-mediated storeoperated $\mathrm{Ca}(2)(+)$ entry in airway smooth muscle cell proliferation. J. Appl. Physiol. (1985) 110, 1256-1263 (2011).

51. Wei, C. et al. Calcium flickers steer cell migration. Nature 457, 901-905 (2009).

52. Martini, F. J. \& Valdeolmillos, M. Actomyosin contraction at the cell rear drives nuclear translocation in migrating cortical interneurons. J. Neurosci. 30, 8660-8670 (2010).

53. Fish, J. E. et al. Dynamic regulation of VEGF-inducible genes by an ERK/ERG/ p300 transcriptional network. Development 144, 2428-2444 (2017).

54. Luca, V. C. et al. Structural basis for Notch1 engagement of Delta-like 4. Science 347, 847-854 (2015).

55. Karkkainen, M. J. et al. Missense mutations interfere with VEGFR-3 signalling in primary lymphoedema. Nat. Genet. 25, 153-159 (2000).

56. Kok, F. O. et al. Reverse genetic screening reveals poor correlation between morpholino-induced and mutant phenotypes in zebrafish. Dev. Cell. 32, 97-108 (2015).

57. Robu, M. E. et al. p53 activation by knockdown technologies. PLoS Genet. 3, e78 (2007).

58. McGlincy, N. J. \& Smith, C. W. J. Alternative splicing resulting in nonsensemediated mRNA decay: what is the meaning of nonsense? Trends Biochem. Sci. 33, 385-393 (2008).

59. Guruharsha, K. G. et al. A protein complex network of Drosophila melanogaster. Cell 147, 690-703 (2011).

60. Wales, P. et al. Calcium-mediated actin reset (CaAR) mediates acute cell adaptations. eLife 5, e19850 (2016)

61. Hartzell, C. A., Jankowska, K. I., Burkhardt, J. K. \& Lewis, R. S. Calcium influx through CRAC channels controls actin organization and dynamics at the immune synapse. eLife 5, e14850 (2016).

62. Noren, D. P. et al. Endothelial cells decode VEGF-mediated Ca2+ signaling patterns to produce distinct functional responses. Sci. Signal. 9, ra20 (2016).

63. Lawson, N. D., Vogel, A. M. \& Weinstein, B. M. Sonic hedgehog and vascular endothelial growth factor act upstream of the Notch pathway during arterial endothelial differentiation. Dev. Cell 3, 127-136 (2002).

64. Bollig, F. et al. A highly conserved retinoic acid responsive element controls wtla expression in the zebrafish pronephros. Development 136, 2883-2892 (2009).

65. Blum, Y. et al. Complex cell rearrangements during intersegmental vessel sprouting and vessel fusion in the zebrafish embryo. Dev. Biol. 316, 312-322, 8 (2008).

66. Chi, N. C. et al. Foxn 4 directly regulates tbx $2 \mathrm{~b}$ expression and atrioventricular canal formation. Genes Dev. 22, 734-739 (2008).

67. Kwan, K. M. et al. The Tol2kit: a multisite gateway-based construction kit for Tol2 transposon transgenesis constructs. Dev. Dyn. 236, 3088-3099 (2007).

68. Zygmunt, T. et al. Semaphorin-PlexinD1 signaling limits angiogenic potential via the VEGF decoy receptor sFlt1. Dev. Cell 21, 301-314 (2011).

69. Riedl, J. et al. Lifeact: a versatile marker to visualize F-actin. Nat. Methods 5, 605-607 (2008).

70. Villefranc, J. A., Amigo, J. \& Lawson, N. D. Gateway compatible vectors for analysis of gene function in the zebrafish. Dev. Dyn. 236, 3077-3087 (2007).

71. Wilkinson, R. N. et al. Hedgehog signaling via a calcitonin receptor-like receptor can induce arterial differentiation independently of VEGF signaling in zebrafish. Blood 120, 477-488 (2012).

72. Ablain, J., Durand, E. M., Yang, S., Zhou, Y. \& Zon, L. I. A CRISPR/Cas9 vector system for tissue-specific gene disruption in zebrafish. Dev. Cell 32, 756-764 (2015).
73. De Angelis, J. E. et al. Tmem2 regulates embryonic Vegf signaling by controlling hyaluronic acid turnover. Dev. Cell 40, 123-136 (2017).

74. Inoue, D. \& Wittbrodt, J. One for all-a highly efficient and versatile method for fluorescent immunostaining in fish embryos. PLoS One 6, e19713 (2011).

\section{Acknowledgements}

We thank E. Honoré for personal communication, helpful discussions and critical reading of the manuscript. We thank D. Stainier for the pT3TS-dCas9 plasmid; K. Kawakami for the pTol2UASGCaMP7a plasmid; E. Markham and K. Plant for technical support; E. Seward and N. Ogryzko for sharing reagents; and K. Fisher for helpful discussions. This work was supported by a JG Graves Medical Research Fellowship awarded to R.N.W., Royal Society Research grant RG120564 awarded to R.N.W., Diabetes UK grant 17/0005678 awarded to R.N.W., BHF Infrastructure Grant IG/15/1/ 31328 awarded to T.J.A.C. and R.N.W., BBSRC grant BB/R015457/1 awarded to F.v.E. and R.N.W., NC3Rs grant NC/P001173/1 awarded to T.J.A.C. and R.N.W., and Wellcome Trust Seed Award (210152/Z/18/Z) to R.B.M. A.M.S. was funded by a Jules Thorne $\mathrm{PhD}$ scholarship awarded to R.N.W. and T.J.A.C.

\section{Author contribution}

R.N.W. conceived the project. A.M.S., F.v.E., A.L.A., H.L.W. and R.N.W. designed experiments. A.M.S., S.K., Y.C., Z.J., R.B.M., H.R.K., K.C., F.V.E. and R.N.W. conducted experiments. A.M.S., S.K., Y.C., Z.J., K.C., R.B.M., A.L.A., H.L.W., F.v.E., T.J.A.C. and R.N.W. analysed data. R.N.W. and A.M.S., aided by the other authors, wrote the paper.

\section{Additional information}

Supplementary Information accompanies this paper at https://doi.org/10.1038/s41467019-08590-7.

Competing interests: The authors declare no competing interests.

Reprints and permission information is available online at http://npg.nature.com/ reprintsandpermissions/

Journal peer review information: Nature Communications thanks the anonymous reviewers for their contribution to the peer review of this work.

Publisher's note: Springer Nature remains neutral with regard to jurisdictional claims in published maps and institutional affiliations.

(c) (i) Open Access This article is licensed under a Creative Commons C. Attribution 4.0 International License, which permits use, sharing, adaptation, distribution and reproduction in any medium or format, as long as you give appropriate credit to the original author(s) and the source, provide a link to the Creative Commons license, and indicate if changes were made. The images or other third party material in this article are included in the article's Creative Commons license, unless indicated otherwise in a credit line to the material. If material is not included in the article's Creative Commons license and your intended use is not permitted by statutory regulation or exceeds the permitted use, you will need to obtain permission directly from the copyright holder. To view a copy of this license, visit http://creativecommons.org/ licenses/by/4.0/.

(C) The Author(s) 2019 